\title{
Stable Multiphase Moving Particle Semi-implicit Method for Incompressible Interfacial Flow
}

\author{
Guangtao Duan $^{a b}$, Bin Chen $^{a *}$, Seiichi Koshizuka ${ }^{b}$, Hao Xiang ${ }^{a}$ \\ ${ }^{a}$ State Key Laboratory of Multiphase Flow in Power Engineering, Xi'an Jiaotong University, Xi'an \\ 710049, PR China \\ ${ }^{b}$ Department of Systems Innovation, the University of Tokyo, 7-3-1, Hongo, Bunkyo-ku, Tokyo
}

113-8656, Japan

\section{ABSTRACT}

The moving particle semi-implicit (MPS) method is extended into a multiphase MPS (MMPS) method, where multiphase fluids are modeled as a multi-viscosity and multi-density fluid. Interparticle viscosity and density are adopted to model the interaction between particles of different phases. However, such a straightforward extension is prone to instability because the light particles at the interface suffer from exceptionally high acceleration. Therefore, two approaches, MMPS-HD (harmonic density) and MMPS-CA (continuous acceleration), are proposed to suppress the instability. In the first approach, harmonic mean interparticle density is applied to discretize the multiphase pressure Poisson equation to avoid the exceptionally high acceleration at the interface. In the second approach, new MMPS formulations are derived from the locally weighted average of interaction acceleration between particles to guarantee the continuity of acceleration and velocity. The particle

*Corresponding author. Tel:+86-29-82667326; Fax:+86-29-82669033.

E-mail address: chenbin@mail.xjtu.edu.cn (B. Chen); koshizuka@sys.t.u-tokyo.ac.jp (S. Koshizuka); ltpeach@stu.xjtu.edu.cn (G.T. Duan); xianghao@stu.xjtu.edu.cn (H, Xiang) 
stabilizing term (PST) is then decoupled from the original pressure gradient model and adopts the single-phase formulation to guarantee stability. The developed multi-viscosity and -density models are verified using the multi-fluid Poiseuille flow and Rayleigh-Taylor instability, respectively. Furthermore, two benchmark cases of rising bubbles in $2 \mathrm{D}$ and $3 \mathrm{D}$ with a wide range of density and viscosity ratios are simulated to demonstrate the capability and robustness of the proposed methods in complex multiphase flows. The proposed method can produce stable and reliable results up to a high density ratio of approximately 1000 and viscosity ratio of approximately 100 .

Keywords: Multiphase moving particle semi-implicit (MMPS) method; Multiphase flow; Multi-viscosity model; Multi-density model; Pressure gradient model; Stability

\section{Introduction}

Multiphase flows with deformable interfaces are encountered in a wide variety of industrial applications. However, predicting such flows is challenging because of the discontinuity of fluid properties and complicated topological changes at the interface. Multiphase/interfacial flow simulation methods can be generally classified into two groups. The first group comprises grid methods, in which the entire flow field is solved on a Eulerian grid. The interface evolution is described using specific capturing or tracking techniques, such as volume of fluid (VOF) [1], level set [2], and front tracking [3] methods. The VOF method requires sophisticated algorithms to 
reconstruct the interface and avoid diffusion across the interface. The level set method also requires complex procedures to reinitialize and advect the level set function, thereby guaranteeing mass conservation. The front tracking maintains a sharp interface using markers to represent and track the interface [4]. However, the implementation of front tracking is difficult. The major difficulty lies in how the markers interact with the stationary grid and in the redistribution of the markers when scattered or concentrated $[4,5]$. For Eulerian grid methods, such difficulty arises from the requirement of the Lagrangian motion of interface to be specifically captured or tracked on a stationary frame.

By contrast, full Lagrangian approaches are considered the second group for solving the flow field and tracking the interface simultaneously. Such mesh-free particle methods include the smoothed particle hydrodynamics (SPH) $[6,7]$ and the moving particle semi-implicit (MPS) [8] methods. SPH was initially designed for astrophysical applications [6, 7], and then extended to solid and fluid mechanics [9], whereas the MPS method was originally designed for incompressible flows [8]. In such methods, each fluid phase is discretized into a particle species with specific physical properties. All particle species interact with one another based on governing equations and move on a Lagrangian frame to represent multiphase flows. Hence, the main advantage of particle methods is that developing interface tracking and reconstruction techniques, such as VOF and level set methods, becomes unnecessary. Specifically, because the advection of a color function is directly and accurately 
calculated by the Lagrangian motion of particles, the sharp jump of a color function is guaranteed, which represents the interface. Therefore, the numerical diffusion problem in advecting a color function, which is typically faced by VOF and level set methods, can be avoided.

Owing to the Lagrangian nature of particle methods, SPH and MPS have been used in free surface flows, where only the liquid phase is solved and the gas phase is ignored. Thus, particle methods should be extended to multiphase solvers for general multiphase flows, where all fluid phases are solved. Currently, most multiphase particle solvers are based on explicit weakly-compressible SPH (WCSPH, such as [10-15]). Several researchers have developed multiphase incompressible SPH (ISPH) methods (e.g., [16-19]) because the semi-implicit/projection algorithm can guarantee improved incompressibility with great potential for treating high density ratio and high Reynolds number flow. However, stability is a critical issue in these methods. According to $\mathrm{Hu}$ and Adams [16], interparticle-averaged quantities are used to model the interaction between different phases. A strict CFL condition, which may result in extremely small time steps, is adopted to stabilize their ISPH simulations. Subsequently, $\mathrm{Hu}$ and Adams [17] alleviated the obstacle of small time steps by developing an exact projection approach, where the divergence and gradient models are directly combined to calculate the Laplacian model. This approach can accurately and consistently predict the variation of particle number density, allowing a precise constant-density constraint to be imposed. Nevertheless, the new complex Laplacian 
model [17] will decrease parallel efficiency and increase computational cost because it refers to more neighbor particles (especially in 3D). In the multiphase ISPH methods by Tofighi and Yildiz [18] and Zainali et al. [19], artificial particle displacement is directly enforced to stabilize simulations. However, artificial adjustment parameters have to be carefully selected to preserve the accuracy of simulated results.

In comparison, MPS [8] is an incompressible semi-implicit particle solver, where pressure is solved using the pressure Poisson equation (PPE). The instability caused by the discontinuity of density in extending MPS to a multiphase particle solver is the most challenging issue. The main instability reason is discussed as follows. Based on Newton's third law or the momentum conservation, the forces between different phase particles are continuous across an interface. However, density is discontinuous at the interface and thus acceleration is also discontinuous, which is detrimental to the stability of simulations. Furthermore, the interaction forces between heavy and light particles are usually very high for the latter. Therefore, the latter will suffer from extremely high accelerations, which can easily cause instability. In this sense, multiphase particle methods seem to be naturally unstable at high density ratios.

The currently available solutions can be generally classified into three groups. In the first group, liquid and gas PPEs are solved separately rather than simultaneously to bypass instability (e.g., [20-23]). Nevertheless, artificial buoyant forces are required in the light phase $[21,23]$, and the corresponding parameter requires tuning 
using simple tests, which may not be suitable for complicated multiphase systems. In the second group, only the liquid phase is solved using MPS and the bubble phase is regarded as a hole (see MPS-MAFL by Yoon et al. [24] and its applications [25-29]) or solved using a mesh method (see the hybrid method by Liu et al. [5]). In this manner, instability is bypassed and the interface is fully tracked by Lagrangian particles. However, the initial pressure inside a bubble is difficult to be arranged consistent with that in real free surfaces because the bubble interface is also treated as a free surface in MPS-MAFL. The disadvantage of the hybrid MPS and FVM methods in [5] is their difficult implementation. In summary, simulating general multiphase flows is difficult for these two groups.

In the third group, researchers (e.g., [30-34]) attempt to attenuate the instability caused by the density discontinuity. Shakibaeinia and Jin [30] developed a multi-viscosity model for MPS but utilized an explicit pressure solver (similar to WCSPH by $\mathrm{Hu} \&$ Adams [10]) to simulate the Rayleigh-Taylor (R-T) and Kelvin-Helmholtz $(\mathrm{K}-\mathrm{H})$ instabilities. Jeong et al. [31] and Khayyer and Gotoh [32] mainly focused on using an accurate first-order pressure gradient model to avoid instability and enhance accuracy. Tsuruta et al. [33] proposed a dynamic stabilizer for the single-phase and multiphase simulations. Tsuruta et al. [34] employed potential particles to prevent voids, which cause instability, among particles upon negative pressure. Nevertheless, the above studies (i.e., [30-34]) belonging to the third group have the following drawbacks: 
(1) Single-phase MPS formulations are directly utilized to simulate multiphase flow in [31-34]. Specifically, the interparticle or inter-grid (i.e., grid face) properties that are widely adopted in multiphase formulations of SPH $[10,16]$ or FVM [4, 35] are not employed. Interparticle properties are essential in modeling the interaction among particles with different viscosities/densities (see Sections 3.1 and 3.2).

(2) To the best of our knowledge, no semi-implicit multi-density model has been proposed for MPS. Shakibaeinia and Jin [30] employed interparticle properties for the viscosity term but explicitly calculated the pressure from an equation of state. No semi-implicit multi-density model was presented in [30], possibly because instability occurs easily, as discussed in Sections 2.2 and 2.3.

(3) The quantitative verification of the multiphase simulations at high density ratio is not presented. The highest density ratio of the multiphase simulations in most of the literature $[30,31,33,34]$ is less than 5. Khayyer and Gotoh [32] presented multiphase results with density ratios of approximately 1000, but their pioneering work mainly investigated the stability of different schemes, and the quantitative comparison they performed was not discussed in detail. This may be because the single-phase MPS formulation in [32] can only accurately simulate multiphase flows with low viscosity/density ratios, as discussed in Sections 3.1 and 3.2.

Thus, the extension of MPS into a semi-implicit multiphase particle solver, 
which is the aim of this study, has been rarely discussed in the literature. The interparticle properties in SPH (e.g., [10-12, 14-16]) can be adopted, but the extension is still not straightforward, considering the difference between SPH and MPS. First, the MPS formulations are based on the relative difference of variables, whereas those of the SPH are based on the absolute sum or mean of variables. Thus, the mean interparticle pressure developed by $\mathrm{Hu}$ and Adams [16] in ISPH cannot be adopted in MPS. Interparticle pressure [16] can calculate a mean pressure close to gas particles, between gas and liquid particles, which is helpful in avoiding the high acceleration of gas particles at the interface, thereby stabilizing the simulations. Second, the strategies for enhancing the stability in SPH and MPS are also different. In SPH, reference/background pressures usually cause errors to pressure gradient models [16, 36]. These errors can drive particles from the dense to the dilute particle region, thereby enhancing the stability [36]. In the multiphase ISPH method [16], an extra time step constrain referring to the background pressure can be adopted to guarantee stability. However, background pressure does not contribute to stability in MPS because the MPS gradient model only depends on relative pressure. Koshizuka et al. [37] coupled a stabilizing term using the minimal pressure among neighbor particles $\left(P_{i, m i n}\right)$ into the pressure gradient model to avoid instability in single-phase MPS. Instability occurs easily in multiphase simulations when the stabilizing term is not carefully handled, as discussed in Section 2.3.2 and [32]. Therefore, MPS faces its own special problems in the extension. The solutions in ISPH may not be applied 
directly. Thus, different solutions should be specially designed for MPS.

In this study, two approaches are proposed to avoid instability considering the aforementioned causes, namely the high accelerations of light particles and the discontinuous accelerations at the interface. One slightly alters the solved pressure field based on the harmonic mean interparticle density to prevent heavy particles from exerting extremely large forces on light particles. In this situation, the high accelerations of light particles will not occur at the interface. Harmonic mean density is also adopted in mesh methods to deal with high density ratios [35], but it is still novel in particle methods. In the second approach, the new formulations that can guarantee continuous acceleration at the interface are derived from the locally weighted average of particle interaction accelerations rather than the forces. The acceleration of pressure gradient forces is discontinuous at the interface in most studies on semi-implicit multiphase particle methods (e.g., [16, 17, 31-34]). Moreover, the application of the stabilizing term using $P_{i, \min }$ is carefully investigated because it can also lead to instability. Therefore, a stabilizing term specifically designed for multiphase simulations is developed.

In summary, the objective of this work is to extend MPS into a multiphase moving particle semi-implicit (MMPS) method by solving the instability arising from the density jump. This paper is organized as follows. The single-phase MPS method is briefly described in Section 2.1. The multi-viscosity and -density models are developed directly based on the interparticle properties in Section 2.2. However, the 
straightforward version of MMPS is unstable. Thus, the stabilizing approaches using harmonic mean density and continuous acceleration are discussed in Sections 2.3.1 and 2.3.2, respectively. In Section 3, the multi-viscosity and -density models are first verified using the multi-fluid Poiseuille flow and the R-T instabilities, respectively. Subsequently, the benchmark rising bubbles with low and high density ratios in 2D [38] and 3D [39] are simulated to verify the proposed methods. Finally, three problems are simulated to show the potential of MMPS in complex multiphase systems.

\section{Numerical methods}

The Lagrangian governing equations for incompressible flow are as follows:

$$
\begin{gathered}
\frac{D \rho}{D t}=-\rho \nabla \cdot \mathbf{u}=0, \\
\frac{D \mathbf{u}}{D t}=-\frac{1}{\rho} \nabla P+\frac{1}{\rho} \nabla \cdot(\mu \nabla \mathbf{u})+\mathbf{g}+\frac{\mathbf{F}_{S}}{\rho},
\end{gathered}
$$

where $\mathbf{u}, P, \mathbf{g}$, and $\mathbf{F}_{S}$ are the velocity, pressure, gravity, and surface tension, respectively. In the multiphase flow, density $\rho$ and dynamic viscosity $\mu$ are constant in each phase but vary sharply at the interface. In MPS, the above equations are discretized by particle interaction models, and a semi-implicit algorithm is adopted [8], in which the viscosity, gravity, and surface tension forces are calculated explicitly and pressure is implicitly solved from a PPE.

\subsection{Single-phase MPS method}


The single-phase MPS method is presented here with some effective improvements. The viscosity term is discretized into:

$$
\left\langle\frac{\mu}{\rho} \nabla^{2} \mathbf{u}\right\rangle_{i}=\frac{\mu_{i}}{\rho_{i}} \frac{2 d}{\lambda n_{0}} \sum_{j \neq i}\left(\mathbf{u}_{j}-\mathbf{u}_{i}\right) w\left(\left|\mathbf{r}_{j}-\mathbf{r}_{i}\right|\right)
$$

where $d$ is the number of space dimensions, $\lambda$ is a constant variance of particle distances, $n_{0}$ is the constant particle number density (PND), and $w$ is the weight function (see details in [8]).

The pressure field is solved from an improved PPE as follows:

$$
\left\langle\frac{1}{\rho} \nabla^{2} P\right\rangle_{i}^{k+1}=(1-\gamma) \frac{1}{\Delta t} \nabla \cdot \mathbf{u}^{*}-\gamma \frac{1}{\Delta t^{2}}\left\{\frac{n^{*}-n_{0}}{n_{0}}\right\}+\alpha \frac{1}{\Delta t^{2}} P_{i}^{k+1}
$$

where $\gamma$ is the relaxation coefficient and $\alpha$ is the artificial compressibility coefficient (e.g., [40-42]). The mixed source term consisting of both the divergence-free and constant-PND conditions is widely applied in MPS (e.g., [32, 41-44]). The divergence of $\mathbf{u}^{*}$ in Eq. (4) is discretized by the high-order source (HS) formulation developed by Khayyer and Gotoh [41]. The left side of PPE (Eq. (4)) is discretized by the Laplacian model, as follows:

$$
\left\langle\frac{1}{\rho} \nabla^{2} P\right\rangle_{i}^{k+1}=\frac{1}{\rho_{i}} \frac{2 d}{n_{0} \lambda} \sum_{j \neq i}\left(P_{j}^{k+1}-P_{i}^{k+1}\right) w\left(\left|\mathbf{r}_{j}-\mathbf{r}_{i}\right|\right) .
$$

Khayyer and Gotoh [32] and Jeong et al. [31] recommended the following first-order Taylor-series consistent pressure gradient model for multiphase simulations:

$$
\left\langle\frac{1}{\rho} \nabla P\right\rangle_{i}=\frac{1}{\rho_{i}} \frac{d}{n_{0}} \sum_{j \neq i}\left\{\frac{\left(P_{j}-P_{i}\right)\left(\mathbf{r}_{j}-\mathbf{r}_{i}\right)}{\left|\mathbf{r}_{j}-\mathbf{r}_{i}\right|^{2}} \mathbf{C}_{i j} w\left(\left|\mathbf{r}_{j}-\mathbf{r}_{i}\right|\right)\right\}
$$


where $\mathbf{C}_{i j}$ is a dimensionless corrective matrix (see [31, 43]). However, Gotoh and Khayyer [45] pointed out recently that the stability of Eq. (6) is generally not guaranteed and recommended the dynamic stabilizer developed by Tsuruta et al. [33]. The stabilizing term proposed by Koshizuka et al. [37] using $P_{i, m i n}$ can also stabilize Eq. (6) (see Fig. 4). If the stabilizing term using $P_{i, \min }$ is adopted, Eq. (6) will be written as

$$
\left\langle\frac{1}{\rho} \nabla P\right\rangle_{i}=\frac{1}{\rho_{i}} \frac{d}{n_{0}} \sum_{j \neq i}\left\{\frac{\left(P_{j}-P_{i, \min }\right)\left(\mathbf{r}_{j}-\mathbf{r}_{i}\right)}{\left|\mathbf{r}_{j}-\mathbf{r}_{i}\right|^{2}} \mathbf{C}_{i j} w\left(\left|\mathbf{r}_{j}-\mathbf{r}_{i}\right|\right)\right\}
$$

where $\mathbf{C}_{i j}$ can still improve the accuracy even though Eq. (7) is not exactly first-order Taylor-series consistent. Considering that Eq. (7) is much simpler than the dynamic stabilizer [33], the former is adopted here. In the next section, the MPS method above will be extended into a multiphase solver (MMPS).

\subsection{Extending MPS into MMPS by multi-viscosity and multi-density models}

At the interface, a particle with viscosity $\mu_{1}$ (or density $\rho_{1}$ ) may interact with particles with viscosity $\mu_{2}$ (or density $\rho_{2}$ ). The multi-viscosity and -density models are derived from the interaction between particles with different properties.

Given that the MPS models are derived from the local weighted average of the interaction between particles, the interparticle properties widely adopted in SPH $[10-12,14-18]$ can be utilized. From this perspective, the extension appears straightforward and simple. However, the instability of this extension is the main challenge. This may be the reason Khayyer and Gotoh [32] focused mainly on the 
stability issue rather than the accuracy at high density ratios.

Shakibaeinia and Jin [30] presented a multi-viscosity model for MPS and argued that the harmonic mean interparticle viscosity is more accurate than the arithmetic mean one. Based on their study, the multi-viscosity model using a Gaussian weight function is as follows:

$$
\left\langle\frac{1}{\rho} \nabla \cdot(\mu \nabla \mathbf{u})\right\rangle_{i}=\frac{18 d}{\rho_{i} r_{e}^{2} n_{G}^{0}} \sum_{j} \frac{2 \mu_{i} \mu_{j}}{\mu_{i}+\mu_{j}}\left(\mathbf{u}_{j}-\mathbf{u}_{i}\right) G\left(\left|\mathbf{r}_{j}-\mathbf{r}_{i}\right|\right) .
$$

where the definition of Gaussian $G$ and $n_{G}{ }^{0}$ can be found in [46]. The Gaussian-based Laplacian model is more accurate in modeling the viscous term because Gaussian is a theoretical solution to the viscous/diffusion equation [46]. In this manner, the interaction between particles with different viscosities can be modeled similar to the interparticle viscosity in SPH (e.g., [10-12, 14-16]).

Next, the semi-implicit multi-density model for MMPS is developed. In a multiphase flow, density varies sharply across the interface. Thus, a multiphase PPE is necessary. Considering that the pressure profile mainly depends on the density field in a multiphase flow, the multi-density model actually corresponds to the PPE discretization. The multiphase PPE can be derived in a manner similar to the single-phase PPE in MPS. The following multiphase PPE is adopted in this study:

$$
\left\langle\nabla \cdot\left(\frac{1}{\rho} \nabla P\right)\right\rangle_{i}^{k+1}=(1-\gamma) \frac{1}{\Delta t} \nabla \cdot \mathbf{u}^{*}-\gamma \frac{1}{\Delta t^{2}}\left\{\frac{n^{*}-n_{0}}{n_{0}}\right\}+\alpha \frac{1}{\Delta t^{2}} P_{i}^{k+1},
$$

where $\rho$ varies sharply across the interface. The left side of Eq. (9) is similar to the viscous term in Eq. (8) (omitting $\rho$ ). Therefore, the multiphase PPE can be discretized 
in a manner similar to that of the multi-viscosity model. The harmonic mean interparticle viscosity corresponds to the arithmetic mean interparticle density, which is more accurate for multiphase PPE, because the role of $1 / \rho$ in PPE corresponds to that of $\mu$ in the viscosity term. Accordingly, the left side of Eq. (9) is discretized by the following multi-density model:

$$
\left\langle\nabla \cdot\left(\frac{1}{\rho} \nabla P\right)\right\rangle_{i}^{k+1}=\frac{2 d}{n_{0} \lambda} \sum_{j \neq i} \frac{2}{\rho_{i}+\rho_{j}}\left(P_{j}^{k+1}-P_{i}^{k+1}\right) w\left(\left|\mathbf{r}_{j}-\mathbf{r}_{i}\right|\right) .
$$

To date, the interactions between particles with different viscosities and densities has been modeled by Eqs. (8) and (10), respectively.

Surface tension is also important in simulating interfacial flow. A contoured continuum surface force (CCSF) model has been developed in the authors' previous work [47] and directly adopted here as follows:

$$
\left\langle\frac{\mathbf{F}_{S}}{\rho}\right\rangle_{i}=\frac{\sigma \kappa \nabla C}{\rho_{i}}
$$

where $\sigma, \kappa$, and $C$ are the surface tension coefficient, local curvature, and color function, respectively. In CCSF, the local curvature $\kappa$ is calculated from the smoothed color function, and thus its accuracy is remarkably improved.

By considering surface tension force and replacing Eqs. (3) and (5) in the single-phase MPS with Eqs. (8) and (10), respectively, MPS can be directly extended to MMPS. However, although the three time-step constraints in Section 2.4 are satisfied, the stability of straightforward MMPS cannot be completely guaranteed, especially at high density ratios. In the multiphase ISPH method, Hu and Adams [16] 
derived an extra time-step constraint that refers to the density ratio based on the reference pressure to guarantee stability. The higher the density ratio is, the smaller the time steps this extra condition produces. However, this condition is impractical for multiphase simulations with high density ratios because of its high computational cost. On the basis of the authors' experience, the time step will become extremely small when the density ratio exceeds 10 .

Next, the stability performance of the straightforward MMPS is investigated. The second bubble rising benchmark problem [38] is simulated to test the stability, considering the high density ratio of 1000:1 and high viscosity ratio of 100:1. The quantitative comparisons of results are discussed in Section 3.3. The simulated results using the pressure gradient models, Eqs. (6) and (7), are respectively presented in Fig. $\mathbf{1}$ (a) and (b), which are unstable at early moments.

Considering that sharply-varied density is the main reason of instability, the following density smoothening scheme is utilized:

$$
\rho_{i}=\frac{1}{\sum_{j \neq i} w_{i j}} \sum_{j \neq i} \rho_{i} w_{i j} .
$$

The corresponding simulated results are shown in Fig. 1 (c) and (d), where instability still occurs early, just slightly later than those in Fig. 1 (a) and (b). Moreover, these simulations are still unstable even with a remarkably reduced time step width. In the following parts, the reasons for instability are analyzed.

Density discontinuity affects the pressure profile in multiphase flows, and thus the pressure terms (i.e., PPE and pressure gradient) cause instability. The 
corresponding acceleration of pressure gradient forces $(\nabla P / \rho)$ to the simulations in Fig. 1 are presented in Fig. 2, where acceleration is extremely high for some gas particles and discontinuous at the interface. When velocity is increased by the acceleration, the large perturbation at the interface causes the instabilities in Fig. 1. To guarantee stability, the extremely high acceleration should be alleviated or removed. Two approaches are proposed to achieve this goal, considering that acceleration refers to both the pressure profiles (i.e., PPE) and pressure gradient models. The first approach modifies PPE to prevent the solved pressure from causing high acceleration at the interface, whereas the second one improves the pressure gradient model to ensure the continuity of the calculated acceleration at the interface. The two approaches are discussed in Section 2.3.

\subsection{Stable MMPS approaches}

\subsubsection{Harmonic mean interparticle density for multiphase PPE}

Pressure distribution is closely related to the density field in multiphase flows. Specifically, pressure is derived from the following discretized multiphase PPE:

$$
\frac{2 d}{n_{0} \lambda} \sum_{j \neq i} \frac{1}{\rho_{i j}}\left(P_{j}-P_{i}\right) w\left(\left|\mathbf{r}_{j}-\mathbf{r}_{i}\right|\right)=\frac{(1-\gamma)}{\Delta t} \nabla \cdot \mathbf{u}^{*}-\frac{\gamma}{\Delta t^{2}}\left\{\frac{n^{*}-n_{0}}{n_{0}}\right\}+\alpha \frac{1}{\Delta t^{2}} P_{i}^{k+1},
$$

where $\rho_{i j}$ is an interparticle density. Note that arithmetic mean interparticle density is used in Eq. (10). Then, we discuss how the density field affects the pressure profile based on Eq. (13). Taking the gas-liquid flow as an example, the magnitudes of the source term in Eq. (13) in both phases are comparable. However, the magnitudes of 
the matrix coefficients of pressure difference differ significantly in the two phases. In the gas phase, the matrix coefficient is extremely high because the interparticle density $\left(\rho_{i j}\right)$ is very low, which results in a slight variation in pressure. By contrast, the matrix coefficient is very low in the liquid phase because $\rho_{i j}$ is high, which results in a significant variation in pressure. The pressure distribution is illustrated in Fig. 3 (a), where pressure varies slightly in gas and sharply in liquid. When the arithmetic mean density scheme is adopted, the pressure profile is accurately calculated, as shown in Fig. 3 (a), where the turning point of pressure profile effectively represents the interface. In Fig. 3 (a), particle $i$ is a gas particle, while one of its neighbor particles, $j$, is a liquid particle. When pressure gradient is calculated, the pressure difference between particles $i$ and $j$ is too high for gas particle $i$, which has a low density. Therefore, gas particle $i$ suffers from extremely high acceleration, as shown in Fig. 2, and blow-up instabilities occur, as shown in Fig. 1. Such instability may be avoided by slightly modifying the pressure distribution in the transition region. Using the harmonic mean interparticle density, the interparticle density in the transition region is always close to the small density (gas density) regardless of the liquid density. Therefore, the pressure distribution in the transition region always varies slightly in a manner similar to that in the gas phase, as shown by the dashed line in Fig. 3 (b). In this situation, the pressure difference in the transition region is not too high to cause a dramatic acceleration in the gas particles, thereby guaranteeing the stability of the simulation. Therefore, the final stable formulation of the left side of the multiphase 
PPE (Eq. (9)) is:

$$
\left\langle\nabla \cdot\left(\frac{1}{\rho} \nabla P\right)\right\rangle_{i}^{k+1}=\frac{2 d}{n_{0} \lambda} \sum_{j \neq i} \frac{\rho_{i}+\rho_{j}}{2 \rho_{i} \rho_{j}}\left(P_{j}^{k+1}-P_{i}^{k+1}\right) w\left(\left|\mathbf{r}_{j}-\mathbf{r}_{i}\right|\right) .
$$

With the harmonic mean interparticle density, the turning point of pressure distribution will not coincide with the phase interface, as shown in Fig. 3 (b). The pressure interface (i.e., turning point) is shifted to the liquid side slightly. Therefore, stability is guaranteed by a slight loss in accuracy. Meanwhile, a fine particle arrangement can reduce the thickness of the transition region, thereby improving the accuracy of the above stable formulation (Eq. (14)).

In this approach, the modification is simple, that is, Eq. (10) is substituted with Eq. (14) in the straightforward MMPS method, but stabilizing simulations in physics becomes possible. Simulated results using different pressure gradient models, Eqs. (6) and (7), are presented in Fig. 4. The simulations by Eq. (14) in Fig. 4 are more stable than the those by Eq. (10) in Fig. 1. In Eq. (6), the simulation can run stably for some time but is easily destabilized (Fig. 4 (a)) because the stabilizing term using $P_{i, \min }$ is not utilized. After Eq. (7) is used, the simulation finally stabilizes, as shown in Fig. 4 (b) and (c). Break up is expected in the test case [38]. However, break up does not occur in Fig. 4 (c) because the resolution $(100 \times 200)$ is not fine enough. In Section 3.3, break up occurs with the finer particle arrangements $(200 \times 400$ or $300 \times 600)$. The pressure profiles of the test case simulated using different interparticle densities are presented in Fig. 5, corresponding to the diagrams in Fig. 3. When an arithmetic mean interparticle density (Eq. (10)) is adopted, pressure is accurate and its turning 
point coincides with the interface, as shown in Fig. 5 (a). In this situation, the gas particles at the interface suffer from extremely high accelerations, as shown in Fig. 2, thereby inducing instability (Fig. 1). When harmonic mean interparticle density is applied in Fig. 5 (b), pressure varies slightly in the transition region and gas particles do not face high pressure variations, thereby guaranteeing stability. When the single-phase formulation (i.e., Eq. (5)) is utilized in Fig. 5 (c), the pressure profile is unreasonable because the variation is also large in the gas phase. Therefore, the pressure interface is unclear (note that the corresponding simulation is also unstable).

This MMPS with harmonic mean interparticle density approach is abbreviated to MMPS-HD for convenience. The overall procedures of MMPS-HD are as follows:

(1) The viscous and surface tension terms are explicitly calculated using Eqs. (8) and (11), respectively.

(2) The pressure field is implicitly solved using Eqs. (9) and (14).

(3) The pressure gradient model is calculated using Eq. (7).

\subsubsection{MMPS formulations with continuous acceleration}

In the above approach, the less accurate harmonic mean interparticle density (i.e., Eq. (14)) is used for PPE to guarantee stability. To enhance accuracy, the arithmetic mean interparticle density (i.e., Eq. (10)) is applied to PPE in this approach, and the pressure gradient model is modified for stability.

From the pressure gradient perspective, discontinuous acceleration is the main cause of instability, as discussed below. In MPS, the pressure gradient force between a 
pair of particles $i$ and $j$ is $\left(P_{j}-P_{i}\right)\left(\mathbf{r}_{j}-\mathbf{r}_{i}\right) /\left|\mathbf{r}_{j}-\mathbf{r}_{i}\right|^{2}$, which is the same for both particles $i$ and $j$, as shown in Fig. 6. Eq. (6) is derived from the local weighted average of pressure gradient forces between particles. If the force is exerted on particles $i$ and $j$ separately, as shown in Eq. (6), the acceleration will be $\left(P_{j}-P_{i}\right)\left(\mathbf{r}_{j}-\mathbf{r}_{i}\right) /\left(\rho_{i}\left|\mathbf{r}_{j}-\mathbf{r}_{i}\right|^{2}\right)$ and $\left(P_{j}-P_{i}\right)\left(\mathbf{r}_{j}-\mathbf{r}_{i}\right) /\left(\rho_{j}\left|\mathbf{r}_{j}-\mathbf{r}_{i}\right|^{2}\right)$, respectively. If particles $i$ and $j$ are in different phases, the above acceleration will be discontinuous. For the example, in Fig. 6, the acceleration of gas particle 2 is 1000 times that of the liquid particle 1, which is detrimental to stability. The discontinuous acceleration of the pressure gradient force is presented in Fig. 2. When velocity is increased by acceleration, a large perturbation of velocity occurs at the interface, leading to instability. However, if the pressure gradient force, $\left(P_{j}-P_{i}\right)\left(\mathbf{r}_{j}-\mathbf{r}_{i}\right) /\left|\mathbf{r}_{j}-\mathbf{r}_{i}\right|^{2}$, is shared by both particles $i$ and $j$, the acceleration will be $2\left(P_{j}-P_{i}\right)\left(\mathbf{r}_{j}-\mathbf{r}_{i}\right) /\left(\left(\rho_{i+} \rho_{j}\right)\left|\mathbf{r}_{j}-\mathbf{r}_{i}\right|^{2}\right)$, which is the same for both particles and continuous at the interface, as shown in Fig. 6. Furthermore, using continuous acceleration to derive MMPS formulations may avoid instability. Meanwhile, the viscous and surface tension forces can produce discontinuous acceleration at the interface from Eqs. (8) and (11), respectively. In the original MPS, the single-phase formulations are derived from the local weighted average of the particle interaction forces. In this approach, deriving new multiphase formulations from the local weighted average of the particle interaction accelerations is necessary to guarantee stability.

To maintain the acceleration, mutual interparticle viscous forces should be divided by the arithmetic mean density between particles $i$ and $j$. Thus, the 
multi-viscosity model with continuous acceleration becomes:

$$
\left\langle\frac{1}{\rho} \nabla \cdot(\mu \nabla \mathbf{u})\right\rangle_{i}=\frac{18 d}{r_{e}^{2} n_{G}^{0}} \sum_{j} \frac{2 \mu_{i} \mu_{j}}{\mu_{i}+\mu_{j}} \frac{2}{\rho_{i}+\rho_{j}}\left(\mathbf{u}_{j}-\mathbf{u}_{i}\right) G\left(\left|\mathbf{r}_{j}-\mathbf{r}_{i}\right|, r_{e}\right) .
$$

To keep the acceleration of the surface tension continuous, the arithmetic mean density across the interface should be utilized. Thus, the acceleration of surface tension is discretized into

$$
\left\langle\frac{\mathbf{F}_{S}}{\rho}\right\rangle_{i}=\frac{2 \sigma \kappa_{i} \nabla C}{\rho_{L}+\rho_{G}}
$$

where $\rho_{L}$ and $\rho_{G}$ are the constant densities (e.g., liquid and gas densities) across the interface. In fact, the acceleration calculated using Eq. (16) is consistent with the formulations in [48, 49], which were developed to maintain the continuous acceleration of surface tension at the interface.

The pressure gradient model based on continuous acceleration is the most difficult. A straightforward extension of Eqs. (6) and (7) can result in the following two models:

$$
\left\langle\frac{1}{\rho} \nabla P\right\rangle_{i}=\frac{d}{n_{0}} \sum_{j \neq i}\left\{\frac{2\left(P_{j}-P_{i}\right)\left(\mathbf{r}_{j}-\mathbf{r}_{i}\right)}{\left(\rho_{i}+\rho_{j}\right)\left|\mathbf{r}_{j}-\mathbf{r}_{i}\right|^{2}} \mathbf{C}_{i j} w_{i j}\right\},
$$

and

$$
\left\langle\frac{1}{\rho} \nabla P\right\rangle_{i}=\frac{d}{n_{0}} \sum_{j \neq i}\left\{\frac{2\left(P_{j}-P_{i, \min }\right)\left(\mathbf{r}_{j}-\mathbf{r}_{i}\right)}{\left(\rho_{i}+\rho_{j}\right)\left|\mathbf{r}_{j}-\mathbf{r}_{i}\right|^{2}} \mathbf{C}_{i j} w_{i j}\right\},
$$

where $w_{i j}$ is the compressed form of $w\left(\left|\mathbf{r}_{j}-\mathbf{r}_{i}\right|\right)$. However, neither can produce stable results, as shown in Fig. 7 (a) and (b). Fig. 7 (a) displays instability because the stabilizing term using $P_{i, \min }$ developed by Koshizuka et al. [37] is not used in Eq. (17). 
However, using $P_{i, \min }$ in the model (i.e., Eq. (18)) causes more instability because the instability in Fig. 7 (b) occurs much earlier than that in Fig. 7 (a). Thus, applying the stabilizing term using $P_{i, \min }$ in Eq. (18) may be inappropriate, thus modifications should be proposed.

First, we analyze how the stabilizing term using $P_{i, \min }$ in [37] works. The basic pressure gradient model in MPS [8] is

$$
\left\langle\frac{1}{\rho} \nabla P\right\rangle_{i}=\frac{1}{\rho_{i}} \frac{d}{n_{0}} \sum_{j \neq i} \frac{\left(P_{j}-P_{i}\right)\left(\mathbf{r}_{j}-\mathbf{r}_{i}\right)}{\left|\mathbf{r}_{j}-\mathbf{r}_{i}\right|^{2}} w_{i j}
$$

However, this model suffers from serious instability, and thus seldom used directly in the literature. The common pressure gradient model is

$$
\left\langle\frac{1}{\rho} \nabla P\right\rangle_{i}=\frac{1}{\rho_{i}} \frac{d}{n_{0}} \sum_{j \neq i} \frac{\left(P_{j}-P_{i, \min }\right)\left(\mathbf{r}_{j}-\mathbf{r}_{i}\right)}{\left|\mathbf{r}_{j}-\mathbf{r}_{i}\right|^{2}} w_{i j},
$$

where $P_{i}$ is replaced by $P_{i, m i n}$, that is, the minimal pressure among neighbor particles [37]. Koshizuka et al. [37] proposed that repulsive forces are guaranteed between particles because $P_{j}-P_{i, \min }$ is always positive, thereby avoiding instability. However, this argument cannot explain the instability in Eq. (18), where repulsive forces are also basically guaranteed. Thus, the reason for the stability of Eq. (20) is investigated in another perspective. Eq. (19) can be separated from Eq. (20) in the following form:

$$
\left\langle\frac{1}{\rho} \nabla P\right\rangle_{i}=\frac{1}{\rho_{i}} \frac{d}{n_{0}} \sum_{j \neq i} \frac{\left(P_{j}-P_{i}\right)\left(\mathbf{r}_{j}-\mathbf{r}_{i}\right)}{\left|\mathbf{r}_{j}-\mathbf{r}_{i}\right|^{2}} w_{i j}+\frac{d}{n_{0}} \sum_{j \neq i} \frac{\left(P_{i}-P_{i, m i n}\right)\left(\mathbf{r}_{j}-\mathbf{r}_{i}\right)}{\rho_{i}\left|\mathbf{r}_{j}-\mathbf{r}_{i}\right|^{2}} w_{i j} .
$$

The second term of RHS in Eq. (21) is essentially the gradient of the constant pressure difference $\left(P_{i}-P_{i, \min }\right)$, which can drive particles from a dense particle region to a dilute one. Thus, it can be regarded as a particle stabilizing term (PST) and its function is 
similar to that of the reference pressure in $\operatorname{SPH}[16,36,50]$. According to $[16,36]$, reference pressure is essential to stabilize SPH especially upon negative pressure. Accordingly, PST is important to maintain the stability of MPS. Both Eqs. (6) and (17) are improved versions of Eq. (19), where PST is not used. Thus, a risk of instability exists when Eq. (6) or (17) is applied (as shown in Fig. 4 (a) and Fig. 7 (a)). Therefore, PST enables Eq. (20) or (21) to produce stable results.

To analyze the reason for the instability of Eq. (18), Eq. (18) is split into two parts, similar to Eq. (21):

$$
\left\langle\frac{1}{\rho} \nabla P\right\rangle_{i}=\frac{d}{n_{0}} \sum_{j \neq i}\left\{\frac{2\left(P_{j}-P_{i}\right)\left(\mathbf{r}_{j}-\mathbf{r}_{i}\right)}{\left(\rho_{i}+\rho_{j}\right)\left|\mathbf{r}_{j}-\mathbf{r}_{i}\right|^{2}} \mathbf{C}_{i j} w_{i j}\right\}+\frac{d}{n_{0}} \sum_{j \neq i}\left\{\frac{2\left(P_{i}-P_{i, m i n}\right)\left(\mathbf{r}_{j}-\mathbf{r}_{i}\right)}{\left(\rho_{i}+\rho_{j}\right)\left|\mathbf{r}_{j}-\mathbf{r}_{i}\right|^{2}} \mathbf{C}_{i j} w_{i j}\right\},
$$

where the second part corresponds to PST. The comparison of the PSTs in Eqs. (21) and (22) show that $\rho_{j}$ is referred to in the latter. Specifically, the term $\left(P_{i}-P_{i, \min }\right) / \rho_{i}$ is a constant in Eq. (21), and thus PST can function normally. However, the term $2\left(P_{j}-P_{i, \min }\right) /\left(\rho_{i}+\rho_{j}\right)$ in Eq. (22) is not constant for all the neighboring particles at the interface. Specifically, the PST in Eq. (22) produces "high pressure" for gas particles and "low pressure" for liquid particles at the interface. Thus, the normal function of PST fails and causes instability in Fig. 7 (b). Therefore, density $\rho_{j}$ should be removed from PST, which should only refer to $\rho_{i}$.

With the above notion, the next tested pressure gradient model is

$$
\left\langle\frac{1}{\rho} \nabla P\right\rangle_{i}=\frac{d}{n_{0}} \sum_{j \neq i}\left\{\frac{2\left(P_{j}-P_{i}\right)\left(\mathbf{r}_{j}-\mathbf{r}_{i}\right)}{\left(\rho_{i}+\rho_{j}\right)\left|\mathbf{r}_{j}-\mathbf{r}_{i}\right|^{2}} \mathbf{C}_{i j} w_{i j}\right\}+\frac{d}{n_{0}} \sum_{j \neq i}\left\{\frac{\left(P_{i}-P_{i, m i n}\right)\left(\mathbf{r}_{j}-\mathbf{r}_{i}\right)}{\rho_{i}\left|\mathbf{r}_{j}-\mathbf{r}_{i}\right|^{2}} \mathbf{C}_{i j} w_{i j}\right\},
$$

where $\rho_{j}$ is no longer referred to in PST. The simulated result is shown in Fig. 7 (c), 
where instability still occurs unexpectedly, but later than in Fig. 7 (b). The problem in the PST of Eq. (23) is subtle because the instability occurs after several hundred time steps. The reason for the instability is discussed below.

When the arithmetic mean interparticle density (i.e., Eq. (10)) is adopted for the multiphase PPE, the pressure differences between the gas and liquid particles are too high for the gas particles and easily cause instability, as discussed in Section 2.3.1. The high pressure difference can cause instability via PST in Eq. (23). The typical simulated pressure profiles of the second benchmark in [38] by the continuous-acceleration formulations and the corresponding contours are shown in Fig. 8, where pressure varies slightly inside the bubble and sharply in the surrounding liquid. Moreover, the pressure inside the bubble is higher than that of the surrounding liquid at the upper interface. For example, particle $i$ is a gas particle and $j$ is a liquid one. If $P_{j}$ is smaller than $P_{i}$, the neighboring minimal pressure of particle $i$ will be $P_{j}$, that is, $P_{i, \min }=P_{j}$. In this situation, $\left(P_{i}-P_{i, \min }\right) / \rho_{i}$ in PST is higher than $2\left(P_{j}-P_{i}\right) /\left(\rho_{i}+\rho_{j}\right)$ in the real pressure gradient part in Eq. (23) because $\rho_{i}$ is much smaller than $\rho_{j}$. Therefore, the gas particles may be over-adjusted by PST. Furthermore, the errors of PST accumulate and cause instability only when the particle distribution becomes slightly irregular. Thus, the instability in Fig. 7 (c) is caused by the accumulated error in several hundred time steps. In short, the incorrect specification of $P_{i_{2} \min }$ mainly causes the instability in Eq. (23).

A new same-phase neighboring minimal pressure marked as $P_{i, \min }^{\prime}$ is proposed 
to avoid the above instability. Specifically, minimal pressure $P_{i, \text { min }}^{\prime}$ is only searched among the neighboring particles that are in the same phase with particle $i$. When $P_{i_{2} \text { min }}$ is replaced by $P_{i, \min }^{\prime}$ in Eq. (23), the final pressure gradient model becomes

$$
\left\langle\frac{1}{\rho} \nabla P\right\rangle_{i}=\frac{d}{n_{0}} \sum_{j \neq i}\left\{\frac{2\left(P_{j}-P_{i}\right)\left(\mathbf{r}_{j}-\mathbf{r}_{i}\right)}{\left(\rho_{i}+\rho_{j}\right)\left|\mathbf{r}_{j}-\mathbf{r}_{i}\right|^{2}} \mathbf{C}_{i j} w_{i j}\right\}+\frac{d}{n_{0}} \sum_{j \neq i}\left\{\frac{\left(P_{i}-P_{i, \min }^{\prime}\right)\left(\mathbf{r}_{j}-\mathbf{r}_{i}\right)}{\rho_{i}\left|\mathbf{r}_{j}-\mathbf{r}_{i}\right|^{2}} \mathbf{C}_{i j} w_{i j}\right\} .
$$

The simulated result, which is stable, is shown in Fig. 7 (d). Although several liquid particles penetrate the bubble, the continuous acceleration between the gas and liquid phases prevents instability. Because density smoothing is applied in the benchmark study [38] and in many other works $[11,30,32,51]$, the density smoothening scheme (i.e., Eq. (12)) is also used, and the corresponding results are presented in Fig. 7 (e) and (f). Break-up occurs at $t=3.0$ without particle penetration, which is consistent with the results in [38], proving that density smoothening can help improve the accuracy of the present method when the density ratio is high.

The reason behind the suppression of the discontinuity of acceleration by the second part of RHS of Eq. (24) (i.e., PST) is discussed here. The magnitudes of the source term in Eq. (13) are comparative in different phases, and thus the ratio, $\left(P_{J}-P_{i}\right) / \rho_{i j}$, has similar magnitudes among different phases. The pressure difference between a pair of particles, $P_{j}-P_{i}$, is basically proportional to their interparticle density $\rho_{i j}$. If a pair of particles are in the same phase, $\rho_{i j}=\rho_{i}$, then the magnitude of $P_{J}-P_{i}$ is similar to that of $P_{i}-P_{i, m i n}^{\prime}$. Thus, the scale of $\left(P_{i}-P_{i, m i n}^{\prime}\right) / \rho_{i}$ is comparable in different phases and helps maintain the continuity of acceleration. Meanwhile, $\left(P_{i}-P_{i_{2} \text { min }}\right) / \rho_{i}$ may be particularly high if the minimal pressure $P_{i, \min }$ comes from a 
different phase. The calculated acceleration by the different parts of the gradient models are presented in Fig. 9. The acceleration of RHS of Eq. (24) is shown in Fig. 9 (a). The contributions of the first (i.e., real gradient) and second (i.e., PST) parts of RHS of Eq. (24) are illustrated in Fig. 9 (b) and (c), respectively. Compared with the discontinuous accelerations in Fig. 2, the accelerations in Fig. 9 can be regarded as continuous. The scales of accelerations of PST are comparable all over the domain after $P_{i, \min }^{\prime}$ is adopted, as shown in Fig. 9 (c). However, if the original $P_{i_{2} \min }$ is applied (i.e., PST of Eq. (23)), exceptionally high gas particle accelerations (similar to Fig. 2) occur at the interface, as shown in Fig. 9 (d). These particularly high accelerations cause instability in Eq. (23), as shown in Fig. 7 (c). Thus, using $P_{i, \min }^{\prime}$ is effective in suppressing the discontinuity of accelerations based on Fig. 9 (c) and (d). In Fig. 9 (e), the irregularity of particle distribution is calculated using

$$
\mathbf{N}_{i}=\frac{d}{n_{0}} \sum_{j \neq i}\left\{\frac{\mathbf{r}_{j}-\mathbf{r}_{i}}{\left|\mathbf{r}_{j}-\mathbf{r}_{i}\right|} w_{i j}\right\} .
$$

The accelerations are concentrated inside and near the bubble in Fig. 9 (a)-(c) because of the high irregularity, as shown in Fig. 9 (e). Therefore, the consistency between $P_{i}-P_{i, \min }^{\prime}$ and $\rho_{i}$ in the PST of Eq. (24) is guaranteed by the introduction of $P_{i, \text { min }}^{\prime}$, which effectively suppresses the discontinuity of acceleration (see Fig. 9 (c) and (d)).

To summarize this approach, the terms referring to fluid properties, such as the viscosity, surface tension, PPE, and real pressure gradient terms, are extended to multiphase formulations based on the local weighted average of the particle 
interaction accelerations. The terms not referring to fluid properties, such as the divergence (of temporary velocity), PND (in PPE source), and PST terms, still adopt the single-phase formulations. In particular, the PST should not be extended into a multiphase formulation, which means (a) PST should be decoupled from the pressure gradient model (the original PST is coupled with the pressure gradient in Eq. (20)), (b) PST should not refer to the densities of different-phase particles, and (c) the minimal pressure $P_{i_{2} \min }$ should not refer to the pressure levels of different-phase particles. If any of the three conditions for the PST is not satisfied, instability will occur easily.

The present (MMPS with continuous acceleration) approach is abbreviated to MMPS-CA for convenience. In summary, the overall procedure of MMPS-CA is as follows:

(1) The viscous and surface tension terms are explicitly calculated using Eqs. (15) and (16) with continuous acceleration, respectively.

(2) The pressure field is solved using Eqs. (9) and (10).

(3) The pressure gradient acceleration is finally calculated using Eq. (24).

If either of the two approaches (MMPS-HD or MMPS-CA) is utilized to simulate the single-phase flow, the whole method will be reduced to the original MPS, as described in Section 2.1. Therefore, MMPS is a mere extension of MPS for multiphase flow and the coding overhead is very small.

\subsection{Time-step constraints and boundary conditions}

After the MMPS-HD and MMPS-CA methods are developed, the following three 
time-step constraints can effectively guarantee the stability. The first constraint is the Courant stability condition (or CFL) $[8,46]$, that is,

$$
u_{\max } \cdot \Delta t \leq C_{0} \cdot l_{0}
$$

where $u_{\max }$ is the maximum velocity and $C_{0}$ is typically 0.2 . The second constraint that refers to the viscosity term [46] is

$$
\frac{v_{\max } \Delta t}{l_{0}^{2}} \leq \frac{k^{2}}{18 d}, \text { where } k=\frac{r_{e}}{l_{0}}
$$

where $v_{\max }$ is the maximum kinematic viscosity in all phases. The third condition for the stability of surface tension $[12,49,52]$ is

$$
\Delta t \leq 0.5 \cdot \sqrt{\frac{\rho l_{0}^{3}}{2 \pi \sigma}} .
$$

Note that (i) the above constraints cannot guarantee the stability of straightforward MMPS, where further time step reduction is necessary at high density ratios, and (ii) the time-step constraint that refers to the reference pressure in ISPH [16] is unnecessary because reference pressure is not used to enhance the stability in MPS. Despite the negative pressure in Fig. 8, the simulation is still stable because PST can function normally in a negative pressure field.

When MPS is extended to MMPS, multiphase boundary conditions become necessary because of the possible intersection of the interface with the free surface or solid wall. In this situation, the multiphase boundary conditions are challenging and the corresponding work is ongoing. In this study, we try to avoid the interaction of the interface with the free surface or solid wall so as to directly implement the 
single-phase boundary conditions. The arrangement of the free surface can be found in [53]. The arrangements of the free- and non-slip boundary conditions are similar to those in $[19,54,55]$. The physical properties (e.g., density or viscosity) of the ghost wall particles are regarded as those of the target particles even when the interface intersects with a solid wall (i.e., single-phase boundary conditions are still directly adopted in this situation). Such an arrangement is simple to implement and effective for stability.

\section{Results and discussions}

In this section, the multi-viscosity and -density models are respectively verified using the multi-fluid Poiseuille flow and the R-T instability. The benchmark bubble rising problems in [38] are then simulated to validate the present method further. Finally, three complex multiphase flows with high density ratios are simulated to show the robustness of the proposed methods. Density smoothening is not applied unless otherwise specified.

\subsection{Multi-fluid Poiseuille flow}

Viscosity forces are dominant in the multi-fluid Poiseuille flow and appropriate for validating the multi-viscosity model. The computational setup is the same with that of [47]. The two fluids adopted have the same density (i.e., 1000). The verification of the multi-viscosity model does not refer to the multi-density model. The viscosity of fluid $1\left(\mu_{1}\right)$ is kept constant at 1000 , whereas that of fluid 2 can be calculated as $\mu_{2}=\mu_{1} / M$, where $M$ is the viscosity ratio. All the adopted variables are 
dimensionless. The analytical solution of the flow can be found in [56]. This test case can help indicate the performance of harmonic and arithmetic multi-density models, between which, the direct comparison is difficult. Specifically, if the density ratio is small, the slight difference in performance between the multi-density models can be easily obscured by the numerical pressure oscillations in MPS [45, 57]. Meanwhile, if the density ratio is high, the direct adoption of the arithmetic multi-density model (i.e., straightforward MMPS) is unstable, and thus comparison is impossible.

First, the performances of different multi-viscosity models were evaluated. The steady velocity profiles with different viscosity ratios $(M)$ in the same particle arrangement (i.e., $50 \times 40$ ) are given in Fig. 10. The harmonic multi-viscosity model corresponds to Eq. (8) or (15). Accordingly, the arithmetic multi-viscosity model refers to the one that uses the arithmetic mean interparticle viscosity. The single-phase viscosity model corresponds to Eq. (3). The mean relative error of the steady velocity is calculated as follows:

$$
\varepsilon_{m}=\frac{1}{N} \sum_{i=1}^{N} \frac{\left|u_{i}-u\left(y_{i}\right)\right|}{u\left(y_{i}\right)},
$$

where $u\left(y_{i}\right)$ is the analytical velocity. The corresponding mean relative errors are summarized in Table 1. According to Table $\mathbf{1}$ and Fig. 10, the harmonic multi-viscosity model produces reasonable and accurate results with different $M$ values. Moreover, results of the arithmetic multi-viscosity model are slightly less accurate than those of the harmonic one but are still reasonable. In Fig. 10 (b) and (c), the turning point of velocity (i.e., velocity interface) is shifted slightly to the less 
viscous side after the arithmetic mean interparticle viscosity is adopted. Furthermore, the relative error caused by the shift increases with $M$. In fact, the shift of velocity interface in Fig. 10 (b) or (c) corresponds to the shift of pressure interface in Fig. 3 (b), and this phenomenon proves the stability in MMPS-HD, as discussed in Section 2.3.1. The single-phase viscosity model produces the least accurate results, which are no longer reasonable when $M$ is higher than 10 because the interparticle viscosity is not adopted in Eq. (3). Considering the similarity between the multi-viscosity and -density models, single-phase models can only be applied to flows with low viscosity and density ratios.

Second, the convergence of different multi-viscosity models is assessed. The relative errors of the steady velocity with $M=50$ at different particle arrangements are shown in Fig. 11, and the corresponding mean relative errors are presented in Table 2. As shown in Fig. 11 (a), the error of the harmonic multi-viscosity model is located mainly near the wall boundary because of the low velocity near the wall. As shown in Fig. 11 (b), the error of the arithmetic multi-viscosity model is near the interface because the velocity interface has slightly shifted, as discussed in the previous paragraph. The extremely high error of the single-phase viscosity model indicates incorrect results, as shown in Fig. 11 (c). Both the harmonic and arithmetic multi-viscosity models are convergent when fine particle arrangements are adopted, as shown in Table 2. However, no convergence tendency was observed in the results of the single-phase viscosity model in Fig. 11 (c) and Table 2. 
In summary, both the harmonic and arithmetic multi-viscosity models are verified using the multi-fluid Poiseuille flow, and the former is more accurate than the latter. The results of the single-phase viscosity model are not practical when the viscosity ratio is high. Moreover, the discretization of multiphase PPE (i.e., Eq. (10) or (14)) is confirmed implicitly because of the similarity between the multi-viscosity and multi-density models. In conclusion, the arithmetic mean interparticle density is more accurate than the harmonic one, and the latter can shift the pressure interface (see Fig. 5 (b)), which stabilizes MMPS-HD. Accordingly, the results of the multiphase flow simulated using the single-phase PPE (i.e., Eq. (5)) will not be accurate when the density ratio is high (see Fig. 5 (c)).

\section{2. $R-T$ instability}

The first test case using R-T instability is similar to the one by Liu et al. [5], which has been used to verify the multiphase simulations by Jeong et al. [31]. In a 1 $\mathrm{m} \times 2 \mathrm{~m}$ tank, heavy fluid with $\rho=3 \mathrm{~kg} \mathrm{~m}^{-3}$ and $v=0.01 \mathrm{~m}^{2} \mathrm{~s}^{-1}$ is poured in the upper half of the tank over a light fluid with $\rho=1 \mathrm{~kg} \mathrm{~m}^{-3}$ and $v=0.01 \mathrm{~m}^{2} \mathrm{~s}^{-1}$, which is poured in the lower half of the tank. Gravity is $10.0 \mathrm{~m} \mathrm{~s}^{-2}$. The downward fluctuation of the interface in the sinusoidal function with an amplitude of $0.06 \mathrm{~m} \mathrm{[5,}$ 31] induces $\mathrm{R}-\mathrm{T}$ instability. The free-slip boundary condition is applied. The snapshots of the simulated R-T by MMPS-CA at different instants are presented in

Fig. 12. The streamlines are continuous at the interface because MMPS-CA produces continuous acceleration. Mushroom caps and vortices can be observed in Fig. 12, 
with the latter causing the former. The shapes of $\mathrm{R}-\mathrm{T}$ are always symmetric, as shown in Fig. 12. However, the streamlines become slightly asymmetric after $t=1.2 \mathrm{~s}$ because of the accumulated errors of pressure oscillation in the particle methods. The streamlines are symmetric at the early stages $(t=0.4$ and $0.8 \mathrm{~s})$. The quantitative evolution of the R-T instability is presented in Fig. 13. A convergence test by MMPS-CA is presented in Fig. 13 (a), where the result of the particle arrangement at $100 \times 200$ is converged. This particle arrangement is used to compare the performance of different methods, as shown in Fig. 13 (b). The simulated results by MMPS-HD and MMPS-CA are similar and in accordance with the literature. However, the results using the single-phase MPS method are inferior to the results by MMPS.

The second test of R-T instability is similar to that of Popinet and Zalesk [58], where density ratio is high. Heavy fluid with $\rho_{h}=1.225 \mathrm{~kg} \mathrm{~m}^{-3}$ and $v_{h}=0.00313 \mathrm{~m}^{2}$ $\mathrm{s}^{-1}$ is placed over a light fluid with $\rho_{l}=0.1694 \mathrm{~kg} \mathrm{~m}^{-3}$ and $v_{l}=0.00313 \mathrm{~m}^{2} \mathrm{~s}^{-1}$ in a 1 $\mathrm{m} \times 4 \mathrm{~m}$ tank, and the free-slip boundary condition is utilized. For the interfacial flow, where surface tension effects are negligible, a spurious fragmentation of interface can occur, as argued by Grenier et al. [11, 12]. To prevent this, slight surface tension $\left(\sigma=2.0 \times 10^{-3} \mathrm{~N} / \mathrm{m}\right)$ is applied in the test case. The grid used in [58] is $64 \times 256$, whereas the present particle arrangement is $100 \times 400$. The comparison between the $\mathrm{R}-\mathrm{T}$ developments simulated by MMPS-HD and the results of front-tracking in [58] are presented in Fig. 14 (a) - (c). Moreover, the simulated R-T shapes are similar to 
the interfaces obtained using front-tracking. Particularly, the positions of the main fronts of $\mathrm{R}-\mathrm{T}$ correspond with the results in [58]. However, the simulation by the single-phase MPS method suffers from instability, as seen in Fig. 14 (d), at $t=0.028 \mathrm{~s}$ because of the high density ratio $\left(\rho_{h} / \rho_{l}=7.23\right)$. The comparison between the results of the MMPS-CA and those in [58] is presented in Fig. 15, where reasonable agreements are observed. Discrepancies are observed near the interaction area of the interface with the solid wall, which are probably caused by the original single-phase boundary condition for the solid wall (see Section 2.4).

From the $\mathrm{R}-\mathrm{T}$ instability simulations, the proposed multi-density models in MMPS-HD and MMPS-CA are proven capable of reasonably predicting the development of $\mathrm{R}-\mathrm{T}$ instability, through which the proposed multi-density model is verified. Moreover, the single-phase MPS method is neither stable nor accurate in the multiphase simulations when the viscosity or density ratio is high (see Fig. 5(c), Fig. 10, Fig. 11(c), and Fig. 14(d)). In the following parts, the single-phase MPS method is not used for comparisons.

\subsection{Comparison with benchmark cases of bubble rising simulations}

Hysing et al. [38] defined two 2D benchmark tests for bubble simulations to verify the multiphase methods quantitatively. These results are reliable because they have been verified and confirmed using different FEM methods. The computational setups of these test cases are described in [38].

In benchmark case 1, a bubble with $R e=35$ and $B o=10$ (termed as $E o$ in [38]) 
rises in a tank, and the density and viscosity ratios are both 10 . The bubble ends up in the ellipsoidal regime, where surface tension is sufficiently strong to hold the bubble without breakup. Shakibaeinia [59] and Zhang et al.[15] simulated the test case using explicit multiphase MPS and WCSPH methods, respectively. The converged grid arrangement is $80 \times 160$ in [38]. Hence, the adopted particle arrangement, that is, 100 $\times 200$, is slightly finer. The artificial compressible coefficient, $\alpha=1.0 \times 10^{-9}$, is appropriate to keep the fluids as incompressible as possible and guarantee the stability of the simulation. The simulated bubble shapes at $t=3$ are compared with the benchmark results in Fig. 16, where good agreements are observed. Moreover, the evolution of the center of mass and rising velocity of the bubble are presented in Fig. 17. The simulated results correspond with those of the benchmark. As shown in Fig. 17 (b), the rising velocity by MMPS-HD suffers more fluctuations (caused by accumulated errors in particle methods) than that by MMPS-CA. Furthermore, the rising velocity simulated by Zhang et al.'s WCSPH method [15] fluctuates even more heavily than the present results, as shown in Fig. 17 (b). In fact, the bubble mean positions simulated by Shakibaeinia [59] are sometimes above and sometimes below the benchmark curve, indicating serious fluctuations in the rising velocity (the results of rising velocity are not presented in [59]). The pressure profiles simulated by MMPS-CA are presented in Fig. 18 at three typical moments. Two reasonable observations are made: (i) the pressure profiles vary slightly inside the bubble because the gas density is small and (ii) the pressure inside the bubbles is higher than that of 
the surrounding liquid because of the strong surface tension. Thus, the present multi-density models (i.e., discretization of multiphase PPE) are reasonable. Initially $(t=0.5)$, the pressure profile is symmetric. However, at later the moments $(t=1.5$ and 3.0), the pressure profile becomes slightly asymmetric (because of the accumulated errors in particle methods), which does not affect the bubble symmetry (see Fig. 16). The solved pressure field may have been high because no free surface is specified in the simulation. The current pressure gradient models in MMPS only depend on the pressure difference. Therefore, the good agreements in Fig. 16 and Fig. 17 verify the MMPS-HD and MMPS-CA methods, and MMPS-CA appears to have produced better results than the MMPS-HD (see Fig. 17 (b)).

In benchmark case 2 [38], the density and viscosity ratios increase to 1000 and 100 , respectively. For this case, $R e=35$ and $B o=125$, hence the bubble is situated somewhere between the skirted and dimpled ellipsoidal cap regimes, indicating a possible breakup. Thus, benchmark case 2 is more challenging than case 1 , as argued by Hysing et al. [38]. To the best of our knowledge, this is the first time that benchmark case 2 is used to verify a multiphase MPS method quantitatively. Sun et al. [60] and Zainali et al. [19] simulated this test case based on WCSPH and ISPH, respectively, to verify the methods they developed. The former presents quantitative results that are used for comparison in this paper. The latter mainly compares the bubble shapes and does not present the quantitative profiles. The quantitative investigation is discussed below. 
First, four different particle arrangements (i.e., $50 \times 100,100 \times 200,200 \times 400$, and $300 \times 600$ ) are used to show the convergence performance of MMPS-HD and MMPS-CA. The $100 \times 200$ arrangement is used to investigate the stability performance of different pressure gradient models in Sections 2.2 and 2.3 (see Fig. 1, Fig. 4, and Fig. 7). Density smoothening is adopted for the convergence test in MMPS-CA and the reason will be discussed in the next paragraph. The simulated bubble shapes at the final moment (i.e., $t=3.0$ ) are presented in Fig. 19 and the corresponding bubble center trajectories are presented in Fig. 20. When the particle resolution is not fine enough (e.g., $50 \times 100$ or $100 \times 200$ ), MMPS-HD fails to predict the breakup behavior of the bubble, whereas MMPS-CA predicts the breakup with long tails (Fig. 19). Moreover, the quantitative results of MMPS-CA are more accurate than those of the MMPS-HD in this situation, as shown in Fig. 20. When the particle resolution is increased (e.g., $200 \times 400$ or $300 \times 600$ ), satellites breaking up from the main bubbles are observed in the results of both MMPS-HD and MMPS-CA (Fig. 19). However, MMPS-HD tends to predict larger satellites than MMPS-CA. Furthermore, the quantitative results of both methods correspond with those in the literature, as shown in Fig. 20. Fig. 19 and Fig. 20 show that the MMPS-CA method can produce better results than MMPS-HD, especially when the particle resolution is coarse. In Fig. 20 (a), the bubble center trajectory simulated by MMPS-HD converges with the benchmark with the increase in resolution. When the resolution is low, the simulated bubble tends to rise faster because the harmonic mean interparticle density 
calculated in the transition region is closer to the gas density, and thus the bubble seems "larger" than its real size in terms of density distribution. In contrast, the simulated bubble by MMPS-CA rises slightly slower than the benchmark with a low resolution (e.g., $50 \times 100$ ). After the particle resolution is increased (e.g., $100 \times 200$ and $200 \times 400$ ), the bubble rose slightly faster than in the literature [38]. When the resolution is fine (e.g., $300 \times 600$ ), the results are close to the benchmark. Therefore, the results of MMPS-CA converge with some fluctuations. Fig. 19 and Fig. 20 show that the results of $200 \times 400$ are similar to those of $300 \times 600$, and thus the former resolution can provide converged results.

Next, the simulated results from the converged $200 \times 400$ are compared with those in the literature. Moreover, the converged grid resolution is $160 \times 320$ in [38]. The comparisons of the mass center and the rising velocity are presented in Fig. 21, and the comparisons of the bubble shapes at $t=3.0$ are illustrated in Fig. 22. After density is smoothened spatially in MMPS-CA, the results of both the rising position and velocity improved. This is the reason why density smoothening is utilized in the above convergence simulations. Furthermore, Zainali et al. [19] applied density smoothening in simulating this problem using their improved ISPH method. The rising velocity simulated by both the present methods and Sun et al.'s WCSPH method [60] suffer some fluctuations that are probably caused by the accumulated errors in the particle methods. Moreover, the second crest of the rising velocity is not obvious in Sun et al.'s results because of the fluctuations, as shown in Fig. 21 (b). 
Furthermore, the rising velocity of MMPS-HD in Fig. 21 (b) suffers more fluctuations than that of MMPS-CA, similar to Fig. 17 (b). Breakup occurs in the present simulations in Fig. 22 and the simulated satellites are in similar positions as those by TP2D [38]. However, MMPS-HD tends to predict larger satellites, whereas MMPS-CA predicts slightly smaller ones. Generally speaking, MMPS-CA is slightly more accurate than MMPS-HD (see Fig. 19, Fig. 20 Fig. 21 (b) and Fig. 22). Therefore, the bubble shapes simulated by MMPS-CA at different moments are compared with those of TP2D, as shown in Fig. 23. Breakup occurs before $t=2.4$. The simulated streamlines at $t=2.1$ (when breakups just begin) are presented in Fig. $\mathbf{2 4}$ to show the reason for the breakup. Two vortices under the bubble are observed and cause the breakup. The profile of the streamlines is slightly asymmetric because of the accumulated errors in the particle methods. From Fig. 20 to Fig. 23, the simulated bubble shapes are generally in good agreement with those in the literature. The above results verify the proposed MMPS-HD and MMPS-CA methods at a density ratio of approximately 1000. Moreover, MMPS-CA with density smoothening can produce more reasonable results than MMPS-HD.

An advantage of the particle methods is the straightforward extension to 3D. After the proposed methods are verified in $2 \mathrm{D}$, the 3D results are discussed below. The 3D simulations of the above benchmark cases in [38] are investigated by Adelsberger et al. [39]. Parallelization is necessary for 3D simulations; however, the CCSF model [47] is not friendly to parallelization because it refers to the neighbor 
particles in $2 \times r_{e}$, which can decrease parallel efficiency and increase overlapping memory. Thus, the reproducing divergence approximation (RDA) in [48] is used to model the surface tension. The verification of RDA can be found in [47, 48]. The grid configuration is $121 \times 241 \times 121$ for both test cases in [39]. In this study, the particle arrangement $80 \times 160 \times 80$ is adopted for benchmark case 1 and $120 \times 240 \times 120$ for benchmark case 2 . Density smoothening is not adopted for benchmark case 1 but utilized for benchmark case 2. The methods, MMPS-HD and MMPS-CA, produce similar results for both test cases. The simulated bubble shapes by MMPS-CA in benchmark case 1 are presented in Fig. 25, which are similar to those in [39]. Stable ellipsoidal shapes are observed because benchmark case 1 ends up in the ellipsoidal regime [38]. The present and literature results are quantitatively compared in Fig. 26, which show a good agreement. The bubble shapes simulated by MMPS-HD for benchmark case 2 are plotted in Fig. 27, where a hollow cap is observed because case 2 is located in the dimpled ellipsoidal-cap regime [38]. Breakup does not occur in the 3D benchmark case 2, as reported in [39]. The mass center and rising velocity in benchmark test 2 in 3D are shown in Fig. 28. The rising velocity is smooth in the 3D illustration because more bubble particles are utilized. Furthermore, the quantitative results by MMPS-CA are slightly better than those of the MMPS-HD in Fig. 26 and Fig. 28. Thus, MMPS-CA is slightly more accurate than MMPS-HD for these benchmark cases. In summary, the proposed MMPS methods are also verified in 3D.

\subsection{Potential of MMPS in complex multiphase flows}


Particle methods have great potential for complex multiphase flows because of their Lagrangian nature. The following three complex multiphase problems are simulated to show the potential of MMPS methods. MMPS-CA is utilized below because it is more accurate.

First, the merging process of two rising bubbles is simulated. The geometric configuration for the problem is shown in Fig. 29. The density and viscosity of the liquid phase are $1000 \mathrm{~kg} / \mathrm{m}^{3}$ and $0.156 \mathrm{~kg} /(\mathrm{m} \cdot \mathrm{s})$, respectively; and those of the bubble phase are $100 \mathrm{~kg} / \mathrm{m}^{3}$ and $0.078 \mathrm{~kg} /(\mathrm{m} \cdot \mathrm{s})$, respectively. The surface tension coefficient $(\sigma)$ is $4.9 \mathrm{~N} / \mathrm{m}$ and the particle arrangement is $500 \times 700$. The above parameters are the same as the ones used in [12]. The more accurate CCSF model in [47] is adopted for surface tension. The comparison between the present results and those by WCSPH and the level set methods [12] are presented in Fig. 30. In general, the simulated merging corresponds with the bubble shapes presented in [12], particularly in the first three snapshots before the breakup of the small lower bubbles. In the fourth snapshot, the simulated breakup behavior is similar to the results of the WCSPH method; however, in the results of the level set method, the satellite bubble breakup from the small bubble remains connected to the small lower bubble. Moreover, the locations and shapes of the largest satellites in the last two snapshots (Fig. 30 (e) and (f)) correspond with those obtained using the WCSPH or the level set methods. The breakup behavior of other small satellite bubbles is difficult to predict accurately because different numerical methods tend to produce slightly different breakup details, 
as argued by Hysing et al. [38]. Therefore, the simulated merging and breakup behaviors correspond well with those of the WCSPH and level set methods, which also verifies the present method.

The second case places the two fluids in benchmark case 2 [38] into a 2D right-angle tube, as shown in Fig. 31 (a) roughly. The liquid and gas phases flow into the tube from the left inlet and flow out from the top outlet. The arrangement of inlet and outlet boundary conditions can be found in [55]. The tube dimensions are comparable with the container size in benchmark case 2 . The width of the tube is 0.5 , which is equal to the diameter of the bubble in benchmark case 2. The horizontal length of the tube is 2.5 and the vertical length is 3.0. Initially, the gas phase is placed in the lower part of the horizontal tube (as shown in Fig. 31 (a) roughly), and the other parts of the right-angle tube are filled with the liquid phase. The inlet velocity of the liquid phase is 4 and that of the gas phase is 1 . The physical parameters of fluids and dimensionless numbers are summarized in Table 3. The Weber number (127.5) describing the ratio of inertial forces to the surface tension effects in this test case is similar to the Bond number (125), which provides the ratio of gravitational forces to the surface tension effects in benchmark case 2. Thus, the breakups during the bubble phase are still expected. A total of 110,000 fluid particles are used and MMPS-CA with density smoothening is applied. In summary, the adopted two fluids are the same as those in benchmark case 2 and the geometric dimensions are comparable. Considering the reasonable results in benchmark case 2 (Fig. 20 to Fig. 23), the 
simulated results in this test case are reasonable.

The simulated snapshots are shown in Fig. 31, where complex breakup behaviors are observed. Initially, the gas phase breaks up into two parts at $t=0.1$ because the liquid phase with high inlet velocity penetrates into the gas phase. The bulk of the initial gas phase rises, deforms, and breaks up from $t=0.2$ to $t=0.75$. At $t=1.0$, a Taylor bubble forms and its top almost reaches the free surface outlet. Subsequently, half of the Taylor bubble flows out of the tube and its tail deforms heavily at $t=1.2$. The initial gas phase just completely flows out of the tube at $t=2.0$. Finally, a steady state similar to Fig. 31 (i) is reached and complex deformation and breakup phenomena are observed. Moreover, the breakup of gas phase mainly happens at the right-angle region, where vortices exist. The advantage of the particle methods for this simulation is that mass conservation is guaranteed for each phase even though complex breakups or coalescence exist, which is challenging for grid methods.

The third one is the R-T instability with a bubble phase, which is similar to the study by $\mathrm{Hu}$ and Adams [17]. The computational setup is similar to the first $\mathrm{R}-\mathrm{T}$ instability discussed in Section 3.2, and the density of the upper heavy phase is still 3 $\mathrm{kg} / \mathrm{m}^{3}$. The lower part of the domain is uniformly filled with original light liquid particles with a density of $1 \mathrm{~kg} / \mathrm{m}^{3}$ and new bubble particles with density of 0.006 $\mathrm{kg} / \mathrm{m}^{3}$. The viscosity of all particles is $0.01 \mathrm{~m}^{2} / \mathrm{s}$. The lower part of the domain is a bubbly region, where the average density is $(1+0.006) / 2=0.503\left(\mathrm{~kg} / \mathrm{m}^{3}\right)$. The highest density ratio in this test is $500: 1$, which is higher than the corresponding ratio (100:1) 
in [17]. The particle arrangement is $100 \times 200$ and the MMPS-CA without density smoothening is used. According to $\mathrm{Hu}$ and Adams [17], this problem is very challenging for Eulerian grid methods. In particle methods, the sharp interface between bubble and liquid particles and the conservation of each phase can be guaranteed. Meanwhile, this problem is challenging for particle methods because particles with high density variances mix with each other and density should not be smoothened spatially. This problem is mainly chosen to show the robustness of the present method because no reference experiment is available, as argued by $\mathrm{Hu}$ and Adams [17]. The simulated positions of particles at different instants are shown in Fig. 32. The interface between the continuous and bubbly phases develops a complex behavior. Moreover, the front of the upper phase reaches the bottom at $t=0.7 \mathrm{~s}$, which is earlier than that in the first $\mathrm{R}-\mathrm{T}$ instability $(t=1.2 \mathrm{~s})$, as shown in Fig. 12 (c). These observations are consistent with those in [17]. Therefore, the results are reasonable.

\section{Concluding remarks}

In this work, the MPS method for incompressible flow is extended to a semi-implicit multiphase particle solver, MMPS. Interparticle viscosity and density are adopted to model the interaction between particles of different phases, and the multi-viscosity and -density models are developed. Instability occurs easily in the straightforward MMPS method because the light particles suffer from particularly high acceleration at the interface. Two methods, MMPS-HD and MMPS-CA are 
proposed to guarantee stability. In MMPS-HD, the harmonic mean interparticle density can slightly shift the pressure interface (i.e., turning point of pressure distribution) to the heavy side to avoid the particularly high acceleration of light particles at the interface. In MMPS-CA, new multiphase formulations are proposed based on the local weighted average of the interaction acceleration (rather than force) between particles. Therefore, the acceleration is continuous at the interface. The following modifications on the pressure gradient model are necessary for the stability of MMPS-CA: (1) the particle stabilizing term (PST) should be decoupled, (2) the density of different-phase particles should not be referred to in PST, and (3) the minimal pressure in PST should only be searched in the same-phase neighbor particles.

Multi-viscosity and -density models are necessary to simulate the multiphase flow, for which the original single-phase MPS method is neither accurate nor stable, especially when the density ratio is high. Therefore, they were initially verified using the multi-fluid Poiseuille flow and the Rayleigh-Taylor instability. The MMPS-HD and MMPS-CA methods are further verified using two benchmark bubble simulations in 2D and 3D. Finally, three challenging multiphase flows are presented to show the potential of MMPS-CA. The results of these show that the proposed methods can produce reliable predictions of incompressible interfacial flow with a wide range of density ratios (from 1 to 1000) and viscosity ratios (from 1 to 100). Moreover, MMPS-CA is slightly more accurate than MMPS-HD. Therefore, MMPS-CA is 
recommended for general multiphase flows.

\section{Acknowledgments}

This research is supported by the National Science Foundation of China (51336006), the Specialized Research Fund for the Doctoral Program of Higher Education (20120201110070), the Fundamental Research Funds for the Central Universities, and the China Scholarship Council (201306280057).

\section{References}

[1] C.W. Hirt, B.D. Nichols, Volume of fluid (VOF) method for the dynamics of free boundaries, J. Comput. Phys., 39 (1981) 201-225.

[2] M. Sussman, P. Smereka, S. Osher, A level set approach for computing solutions to incompressible two-phase flow, J. Comput. Phys., 114 (1994) 146-159.

[3] S.O. Unverdi, G. Tryggvason, A front-tracking method for viscous, incompressible, multi-fluid flows, J. Comput. Phys., 100 (1992) 25-37.

[4] G. Tryggvason, R. Scardovelli, Direct numerical simulations of gas-liquid multiphase flows, Cambridge University Press, 2011.

[5] J. Liu, S. Koshizuka, Y. Oka, A hybrid particle-mesh method for viscous, incompressible, multiphase flows, J. Comput. Phys., 202 (2005) 65-93.

[6] R.A. Gingold, J.J. Monaghan, Smoothed particle hydrodynamics-theory and application to non-spherical stars, Mon. Not. R. Astron. Soc., 181 (1977) 375-389.

[7] L.B. Lucy, A numerical approach to the testing of the fission hypothesis, Astron. J., 82 (1977) 1013-1024.

[8] S. Koshizuka, Y. Oka, Moving-particle semi-implicit method for fragmentation of incompressible fluid, Nucl. Sci. Eng., 123 (1996) 421-434.

[9] J.J. Monaghan, Smoothed particle hydrodynamics, Annu. rev. astron. astrophys., 30 (1992) 543-574.

[10] X. Hu, N. Adams, A multi-phase SPH method for macroscopic and mesoscopic flows, J. Comput. Phys., 213 (2006) 844-861.

[11] N. Grenier, M. Antuono, A. Colagrossi, D. Le Touzé, B. Alessandrini, An Hamiltonian interface SPH formulation for multi-fluid and free surface flows, J. Comput. Phys., 228 (2009) 8380-8393.

[12] N. Grenier, D. Le Touzé, A. Colagrossi, M. Antuono, G. Colicchio, Viscous bubbly flows simulation with an interface SPH model, Ocean. Eng., 69 (2013) 88-102. 
[13] K. Szewc, J. Pozorski, J.-P. Minier, Simulations of single bubbles rising through viscous liquids using smoothed particle hydrodynamics, Int. J. Multiphase Flow, 50 (2013) 98-105.

[14] Z. Chen, Z. Zong, M. Liu, L. Zou, H. Li, C. Shu, An SPH model for multiphase flows with complex interfaces and large density differences, J. Comput. Phys., 283 (2015) 169-188.

[15] A. Zhang, P. Sun, F. Ming, An SPH modeling of bubble rising and coalescing in three dimensions, Comput. Method. Appl. M., 294 (2015) 189-209.

[16] X. Hu, N. Adams, An incompressible multi-phase SPH method, J. Comput. Phys., 227 (2007) 264-278.

[17] X. Hu, N. Adams, A constant-density approach for incompressible multi-phase SPH, J. Comput. Phys., 228 (2009) 2082-2091.

[18] N. Tofighi, M. Yildiz, Numerical simulation of single droplet dynamics in three-phase flows using ISPH, Comput. Math. Appl., 66 (2013) 525-536.

[19] A. Zainali, N. Tofighi, M.S. Shadloo, M. Yildiz, Numerical investigation of Newtonian and non-Newtonian multiphase flows using ISPH method, Comput. Method. Appl. M., 254 (2013) 99-113.

[20] S. Koshizuka, H. Ikeda, Y. Oka, Numerical analysis of fragmentation mechanisms in vapor explosions, Nucl. Eng. Des., 189 (1999) 423-433.

[21] N. Shirakawa, Y. Yamamoto, H. Horie, S. Tsunoyama, Analysis of flows around a BWR spacer by the two-fluid particle interaction method, J. Nucl. Sci. Technol., 39 (2002) 572-581.

[22] H. Gotoh, T. Sakai, Key issues in the particle method for computation of wave breaking, Coast. Eng., 53 (2006) 171-179.

[23] K.S. Kim, M.H. Kim, J.-C. Park, Dynamic coupling between ship motion and three-layer-liquid separator by using moving particle simulation, International Journal of Offshore and Polar Engineering, 24 (2014) 122-128.

[24] H.Y. Yoon, S. Koshizuka, Y. Oka, Direct calculation of bubble growth, departure, and rise in nucleate pool boiling, Int. J. Multiphase Flow, 27 (2001) 277-298.

[25] S. Heo, S. Koshizuka, Y. Oka, Numerical analysis of boiling on high heat-flux and high subcooling condition using MPS-MAFL, Int. J. Heat Mass Transfer, 45 (2002) 2633-2642.

[26] W. Tian, Y. Ishiwatari, S. Ikejiri, M. Yamakawa, Y. Oka, Numerical simulation on void bubble dynamics using moving particle semi-implicit method, Nucl. Eng. Des., 239 (2009) 2382-2390.

[27] W. Tian, Y. Ishiwatari, S. Ikejiri, M. Yamakawa, Y. Oka, Numerical computation of thermally controlled steam bubble condensation using Moving Particle Semi-implicit (MPS) method, Ann. Nucl. Energy, 37 (2010) 5-15.

[28] R. Chen, W. Tian, G. Su, S. Qiu, Y. Ishiwatari, Y. Oka, Numerical investigation on coalescence of bubble pairs rising in a stagnant liquid, Chem. Eng. Sci., 66 (2011) 5055-5063.

[29] X. Li, W. Tian, R. Chen, G. Su, S. Qiu, Numerical simulation on single Taylor 
bubble rising in LBE using moving particle method, Nucl. Eng. Des., 256 (2013) 227-234.

[30] A. Shakibaeinia, Y.-C. Jin, MPS mesh-free particle method for multiphase flows, Comput. Method. Appl. M., 229 (2012) 13-26.

[31] S.-M. Jeong, J.-W. Nam, S.-C. Hwang, J.-C. Park, M.-H. Kim, Numerical prediction of oil amount leaked from a damaged tank using two-dimensional moving particle simulation method, Ocean. Eng., 69 (2013) 70-78.

[32] A. Khayyer, H. Gotoh, Enhancement of performance and stability of MPS mesh-free particle method for multiphase flows characterized by high density ratios, J. Comput. Phys., 242 (2013) 211-233.

[33] N. Tsuruta, A. Khayyer, H. Gotoh, A short note on dynamic stabilization of moving particle semi-implicit method, Comput. Fluids., 82 (2013) 158-164.

[34] N. Tsuruta, A. Khayyer, H. Gotoh, Space potential particles to enhance the stability of projection-based particle methods, International Journal of Computational Fluid Dynamics, 29 (2015) 100-119.

[35] F. Denner, B.G. van Wachem, Fully-Coupled Balanced-Force VOF Framework for Arbitrary Meshes with Least-Squares Curvature Evaluation from Volume Fractions, Numer. Heat. Tr. B-Fund., 65 (2014) 218-255.

[36] S. Adami, X.Y. Hu, N.A. Adams, A transport-velocity formulation for smoothed particle hydrodynamics, J. Comput. Phys., 241 (2013) 292-307.

[37] S. Koshizuka, A. Nobe, Y. Oka, Numerical analysis of breaking waves using the moving particle semi-implicit method, Int. J. Numer. Meth. Fl., 26 (1998) 751-769.

[38] S. Hysing, S. Turek, D. Kuzmin, N. Parolini, E. Burman, S. Ganesan, L. Tobiska, Quantitative benchmark computations of two - dimensional bubble dynamics, Int. J. Numer. Meth. Fl., 60 (2009) 1259-1288.

[39] J. Adelsberger, P. Esser, M. Griebel, S. Groß, M. Klitz, A. Rüttgers, 3D incompressible two-phase flow benchmark computations for rising droplets, in: Proceedings of the 11th World Congress on Computational Mechanics (WCCM XI), Barcelona, Spain, 2014.

[40] H. Ikeda, S. Koshizuka, Y. Oka, H.S. Park, J. Sugimoto, Numerical analysis of jet injection behavior for fuel-coolant interaction using particle method, J. Nucl. Sci. Technol., 38 (2001) 174-182.

[41] A. Khayyer, H. Gotoh, Modified moving particle semi-implicit methods for the prediction of 2D wave impact pressure, Coast. Eng., 56 (2009) 419-440.

[42] M. Tanaka, T. Masunaga, Stabilization and smoothing of pressure in MPS method by quasi-compressibility, J. Comput. Phys., 229 (2010) 4279-4290.

[43] A. Khayyer, H. Gotoh, Enhancement of stability and accuracy of the moving particle semi-implicit method, J. Comput. Phys., 230 (2011) 3093-3118.

[44] M. Kondo, S. Koshizuka, Improvement of stability in moving particle semi implicit method, Int. J. Numer. Meth. Fl., 65 (2011) 638-654.

[45] H. Gotoh, A. Khayyer, Current achievements and future perspectives for projection-based particle methods with applications in ocean engineering, Journal of 
Ocean Engineering and Marine Energy, (2016) 1-28.

[46] G.T. Duan, B. Chen, Stability and accuracy analysis for viscous flow simulation by moving particle semi-implicit method, Fluid Dynamics Research, 45 (2013) 1-15.

[47] G.T. Duan, S. Koshizuka, B. Chen, A contoured continuum surface force model for particle methods, J. Comput. Phys., 298 (2015) 280-304.

[48] S. Adami, X. Hu, N. Adams, A new surface-tension formulation for multi-phase SPH using a reproducing divergence approximation, J. Comput. Phys., 229 (2010) 5011-5021.

[49] J. Brackbill, D.B. Kothe, C. Zemach, A continuum method for modeling surface tension, J. Comput. Phys., 100 (1992) 335-354.

[50] S.J. Lind, R. Xu, P.K. Stansby, B.D. Rogers, Incompressible smoothed particle hydrodynamics for free-surface flows: A generalised diffusion-based algorithm for stability and validations for impulsive flows and propagating waves, J. Comput. Phys., 231 (2012) 1499-1523.

[51] A. Colagrossi, M. Landrini, Numerical simulation of interfacial flows by smoothed particle hydrodynamics, J. Comput. Phys., 191 (2003) 448-475.

[52] J.P. Morris, Simulating surface tension with smoothed particle hydrodynamics, Int. J. Numer. Meth. Fl., 33 (2000) 333-353.

[53] B.H. Lee, J.C. Park, M.H. Kim, S.C. Hwang, Step-by-step improvement of MPS method in simulating violent free-surface motions and impact-loads, Comput. Method. Appl. M., 200 (2011) 1113-1125.

[54] S.J. Cummins, M. Rudman, An SPH projection method, J. Comput. Phys., 152 (1999) 584-607.

[55] G.T. Duan, B. Chen, Large Eddy Simulation by particle method coupled with Sub-Particle-Scale model and application to mixing layer flow, Appl. Math. Model., 39 (2015) 3135-3149.

[56] Q. Cao, K. Sarkar, A.K. Prasad, Direct numerical simulations of two-layer viscosity-stratified flow, Int. J. Multiphase Flow, 30 (2004) 1485-1508.

[57] S. Koshizuka, Current achievements and future perspectives on particle simulation technologies for fluid dynamics and heat transfer, J. Nucl. Sci. Technol., 48 (2011) 155-168.

[58] S. Popinet, S. Zaleski, A front - tracking algorithm for accurate representation of surface tension, Int. J. Numer. Meth. Fl., 30 (1999) 775-793.

[59] A. Shakibaeinia, Mesh-free particle modelling of air-water interaction, in: The 36th IAHR World Congress, Hague, Netherlands, 2015.

[60] P. Sun, F. Ming, A. Zhang, X. Yao, Investigation of coalescing and bouncing of rising bubbles under the wake influences using SPH method, in: ASME 2014 33rd International Conference on Ocean, Offshore and Arctic Engineering, American Society of Mechanical Engineers, 2014. 


\section{Tables captions:}

\section{Table 1}

Mean relative errors of steady velocity with different viscosity ratios $(M)$ in the multi-fluid Poiseuille flow.

\section{Table 2}

Mean relative errors of steady velocity with different particle arrangements and convergence orders in the multi-fluid Poiseuille flow.

\section{Table 3}

Fluid parameters and dimensionless numbers for the gas-liquid two phase flow in a right-angle tube. 


\section{Tables:}

\section{Table 1}

Mean relative errors of steady velocity with different viscosity ratios $(M)$ in the multi-fluid Poiseuille flow.

\begin{tabular}{ccccc}
\hline Mean relative error & $\boldsymbol{M = 2}$ & $\boldsymbol{M}=\mathbf{1 0}$ & $\boldsymbol{M}=\mathbf{2 0}$ & $\boldsymbol{M = 1 0 0}$ \\
\hline Harmonic & $1.40 \%$ & $1.37 \%$ & $1.33 \%$ & $1.32 \%$ \\
Arithmetic & $1.39 \%$ & $2.51 \%$ & $4.36 \%$ & $9.84 \%$ \\
Single-phase & $9.66 \%$ & $113 \%$ & $228 \%$ & $1050 \%$ \\
\hline
\end{tabular}




\section{Table 2}

Mean relative errors of steady velocity with different particle arrangements and convergence orders in the multi-fluid Poiseuille flow.

\begin{tabular}{cccccc}
\hline Mean relative error & $\mathbf{2 5} \times \mathbf{2 0}$ & $\mathbf{5 0} \times \mathbf{4 0}$ & $\mathbf{1 0 0} \times \mathbf{8 0}$ & $\mathbf{2 0 0} \times \mathbf{1 6 0}$ & Convergence order \\
\hline Harmonic & $2.22 \%$ & $1.31 \%$ & $0.85 \%$ & $0.58 \%$ & 0.66 \\
Arithmetic & $12.69 \%$ & $7.5 \%$ & $4.19 \%$ & $2.25 \%$ & 0.86 \\
Single-phase & $527 \%$ & $545 \%$ & $555 \%$ & $560 \%$ & -0.03 \\
\hline
\end{tabular}


Table 3

Fluid parameters and dimensionless numbers for the gas-liquid two phase flow in a right-angle tube.

\begin{tabular}{ccccccccc}
\hline & $\rho$ & $\mu$ & $\boldsymbol{g}$ & $\sigma$ & $R e$ & $W e$ & $\rho / \rho_{g}$ & $\mu_{/} / \mu_{g}$ \\
\hline Liquid & 1000 & 10 & & & 100 & & & \\
Gas & 1 & 0.1 & & & 2.5 & 127.5 & 1000 & 100 \\
\hline
\end{tabular}




\section{Figures Captions:}

Fig. 1. Stability test of the simulated bubble rising problem (density ratio=1000:1) by different schemes using the straightforward MMPS method.

Fig. 2. The pressure gradient acceleration simulated by different schemes using the straightforward MMPS method. The dashed circle is the interface.

Fig. 3. Pressure profiles near the interface in the gas-liquid two-phase flow: (a) the arithmetic mean density scheme and (b) the arithmetic mean density scheme (solid line) and the harmonic mean density scheme (dashed line).

Fig. 4. Stability test of the simulated bubble rising problem (density ratio=1000:1) by different pressure gradient models using the MMPS method with harmonic mean interparticle density.

Fig. 5. Pressure profiles of bubble rising problem by MMPS using: (a) arithmetic mean interparticle density, (b) harmonic mean interparticle density and (c) single-phase PPE formulation. The circles are bubble interface.

Fig. 6. Sketch for pressure gradient forces and corresponding accelerations of two particles (1 and 2) in different phases by different discretizing formulations.

Fig. 7. Stability test of the simulated bubble rising problem (density ratio=1000:1) by different pressure gradient models using the MMPS method based on continuous acceleration.

Fig. 8. Pressure profile of the simulated bubble rising problem (density ratio=1000:1) at $t=0.001 \mathrm{~s}$. The curves are contours of pressure.

Fig. 9. The calculated accelerations by the different parts of pressure gradient models 
at $t=1.5 \mathrm{~s}$.

Fig. 10. Steady velocity profiles by different multi-viscosity models with the viscosity ratio of (a) $M=2$, (b) $M=20$ and (c) $M=100$.

Fig. 11. Relative errors of steady velocity profiles with different particle distances of: (a) the harmonic multi-viscosity model (i.e. Eq. (8)), (b) arithmetic multi-viscosity model and (c) single-phase viscosity model (i.e. Eq. (3)).

Fig. 12. Snapshots of $\mathrm{R}-\mathrm{T}$ instability with streamlines at different moments by MMPS-CA (the particle arrangement is $200 \times 400$ ).

Fig. 13. Evolution of R-T instability: (a) convergence test; (b) comparison of different methods.

Fig. 14. Comparison of $\mathrm{R}-\mathrm{T}$ instability evolutions at different moments. The solid lines are interfaces simulated by front-tracking [58]. Figures (a) - (c) are the results simulated by MMPS-HD. Figure (d) is the result simulated by the single-phase MPS method (instability happens at $t=0.028 \mathrm{~s}$ ).

Fig. 15. Comparison of R-T instability evolutions simulated by MMPS-CA at different moments. The solid lines are interfaces simulated by front-tracking [58].

Fig. 16. Comparison of bubble shapes at $t=3$ for benchmark case 1 in $2 \mathrm{D}$.

Fig. 17. Comparison of (a) centers of mass and (b) rising velocities for benchmark case 1 in $2 \mathrm{D}$.

Fig. 18. The pressure profiles simulated by MMPS-CA at three typical moments in benchmark case 1 . The curve indicates the bubble shape. 
Fig. 19. Snapshots of bubble shapes simulated using different particle resolutions.

Fig. 20. Simulated evolution of centers of mass with different particle resolutions using (a) MMPS-HD and (b) MMPS-CA.

Fig. 21. Comparison of (a) centers of mass and (b) rising velocities for benchmark case 2 where density ratio $=1000$ and viscosity ratio $=100$.

Fig. 22. Comparison of bubble shapes at $t=3$ for benchmark case 2 .

Fig. 23. Comparison between simulated bubble shapes by MMPS-CA and results by TP2D (red curves) in [38] at different moments for benchmark case 2.

Fig. 24. Streamlines of the simulated benchmark case 2 by MMPS-CA at $t=2.1$.

Fig. 25. Bubble shapes simulated by MMPS-CA for benchmark case 1 in 3D.

Fig. 26. Comparison of (a) centers of mass and (b) rising velocities for benchmark case 1 in $3 \mathrm{D}$.

Fig. 27. Bubble shapes simulated by MMPS-HD for benchmark case 1 in 3D.

Fig. 28. Comparison of (a) centers of mass and (b) rising velocities for benchmark case 2 in $3 \mathrm{D}$.

Fig. 29. Diagram of the merging of two bubbles.

Fig. 30. Comparison of two bubbles merging with literature results [12] at different moments: (a) $t(g / R)^{1 / 2}=0.63$; (b) $t(g / R)^{1 / 2}=1.27$; (c) $t(g / R)^{1 / 2}=1.90$; (d) $t(g / R)^{1 / 2}=$ 2.53 ; (e) $t(g / R)^{1 / 2}=2.85$; (f) $t(g / R)^{1 / 2}=3.16$.

Fig. 31. Snapshots of simulated bubble rising in a right-angle tube with complex breakup behaviors (density ratio $=1000$; viscosity ratio $=100$ ) 
Fig. 32. R-T instability of a continuous phase and a bubbly phase at three selected moments (the largest density ratio is 500 and no density smoothening). 


\section{Figures:}
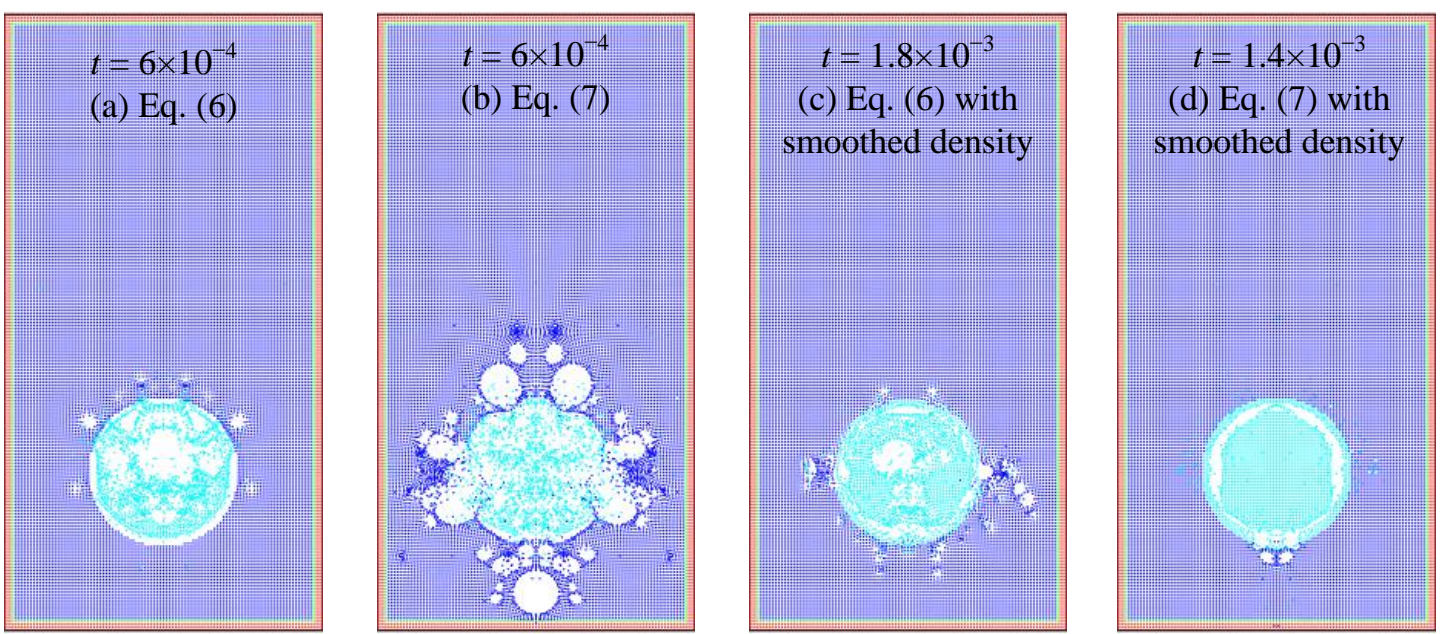

Fig. 1. Stability test of the simulated bubble rising problem (density ratio=1000:1) by different schemes using the straightforward MMPS method. 


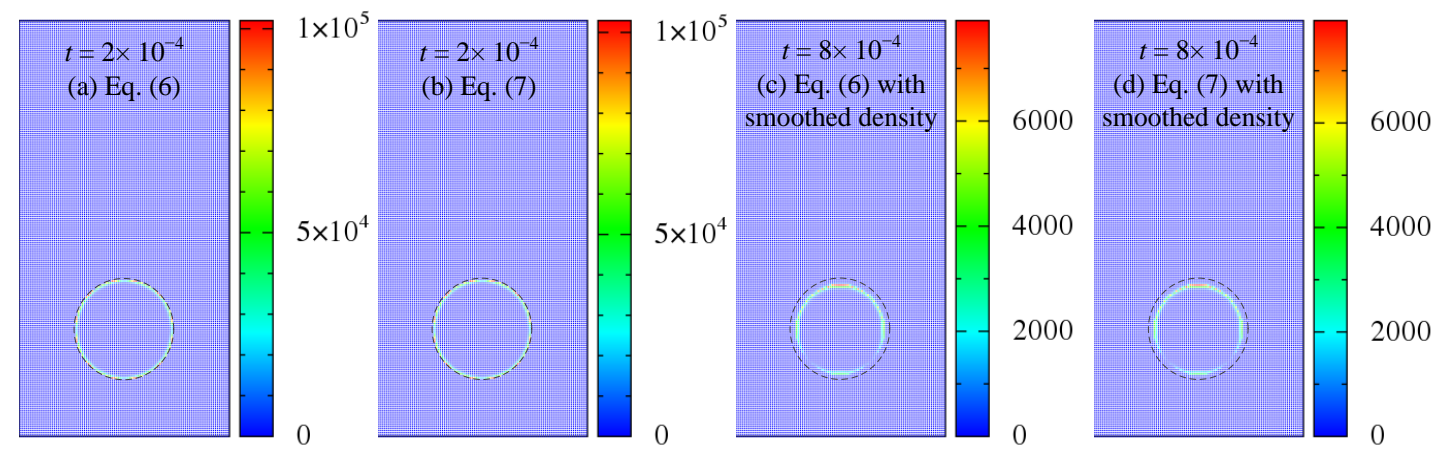

Fig. 2. The pressure gradient acceleration simulated by different schemes using the straightforward MMPS method. The dashed circle is the interface. 

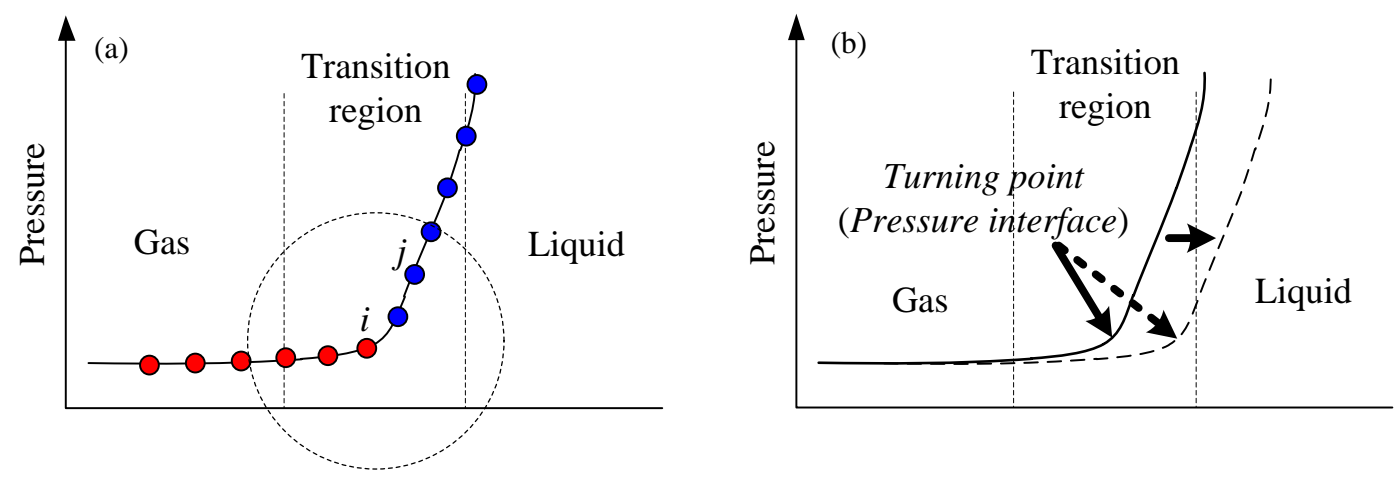

Fig. 3. Pressure profiles near the interface in the gas-liquid two-phase flow: (a) the arithmetic mean density scheme and (b) the arithmetic mean density scheme (solid line) and the harmonic mean density scheme (dashed line). 

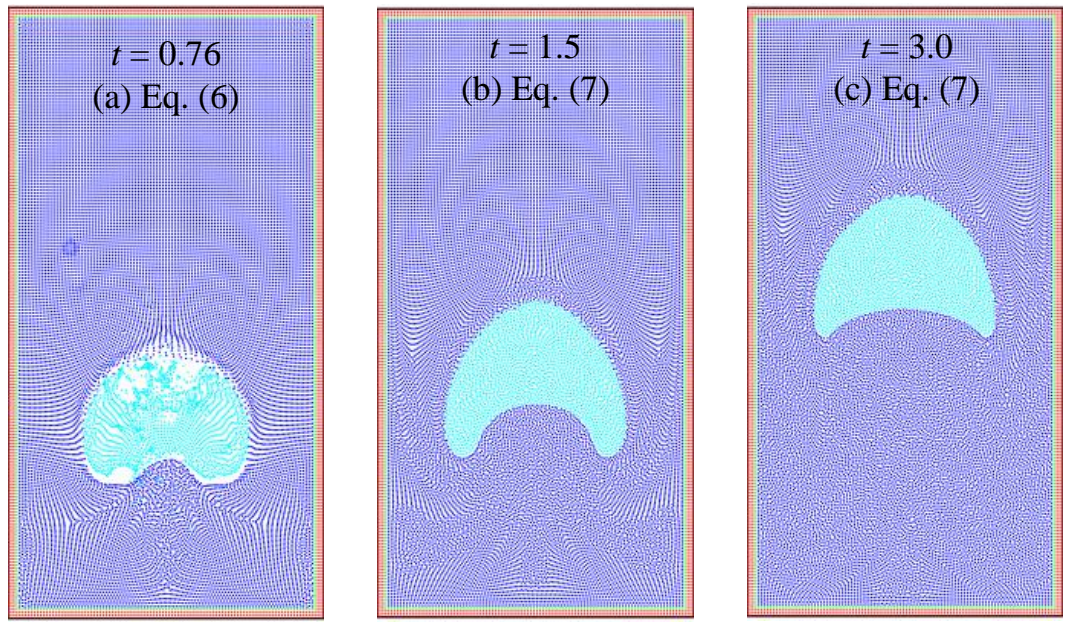

Fig. 4. Stability test of the simulated bubble rising problem (density ratio=1000:1) by different pressure gradient models using the MMPS method with harmonic mean interparticle density. 

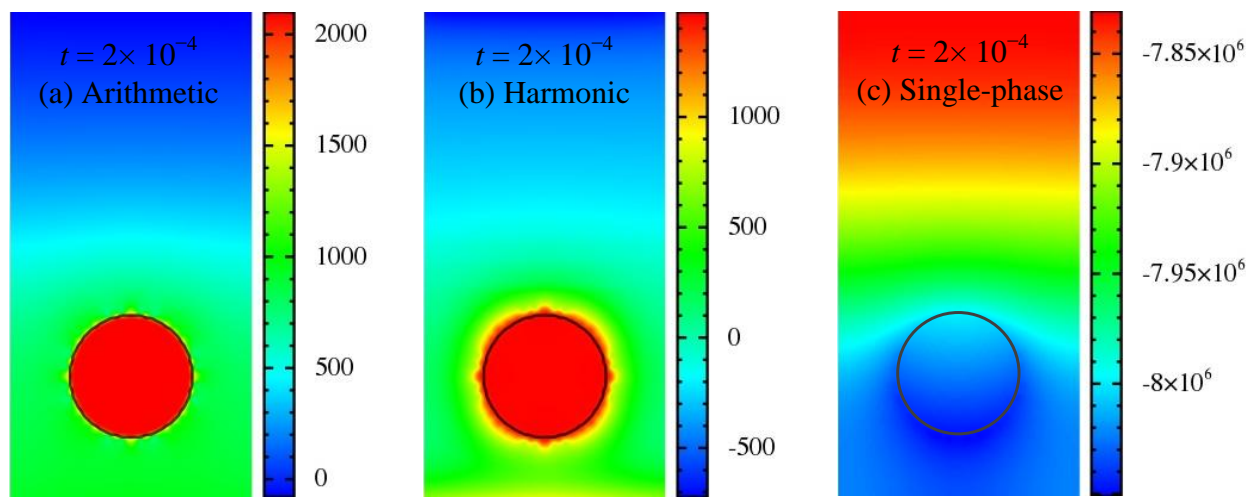

Fig. 5. Pressure profiles of bubble rising problem by MMPS using: (a) arithmetic mean interparticle density, (b) harmonic mean interparticle density and (c) single-phase PPE formulation. The circles are bubble interface. 


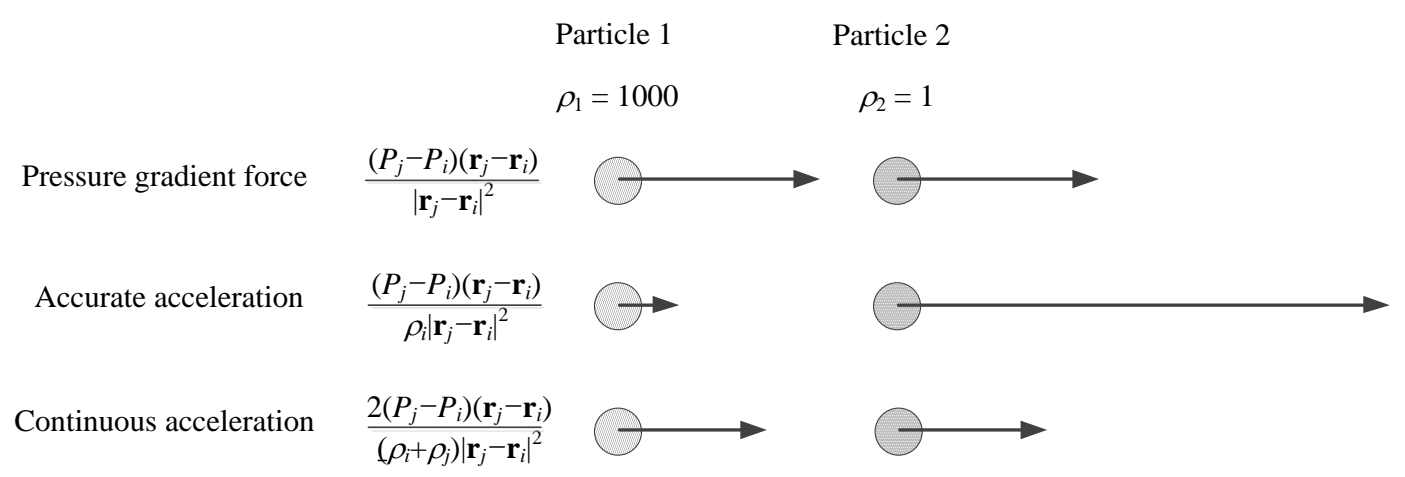

Fig. 6. Sketch for pressure gradient forces and corresponding accelerations of two particles (1 and 2) in different phases by different discretizing formulations. 

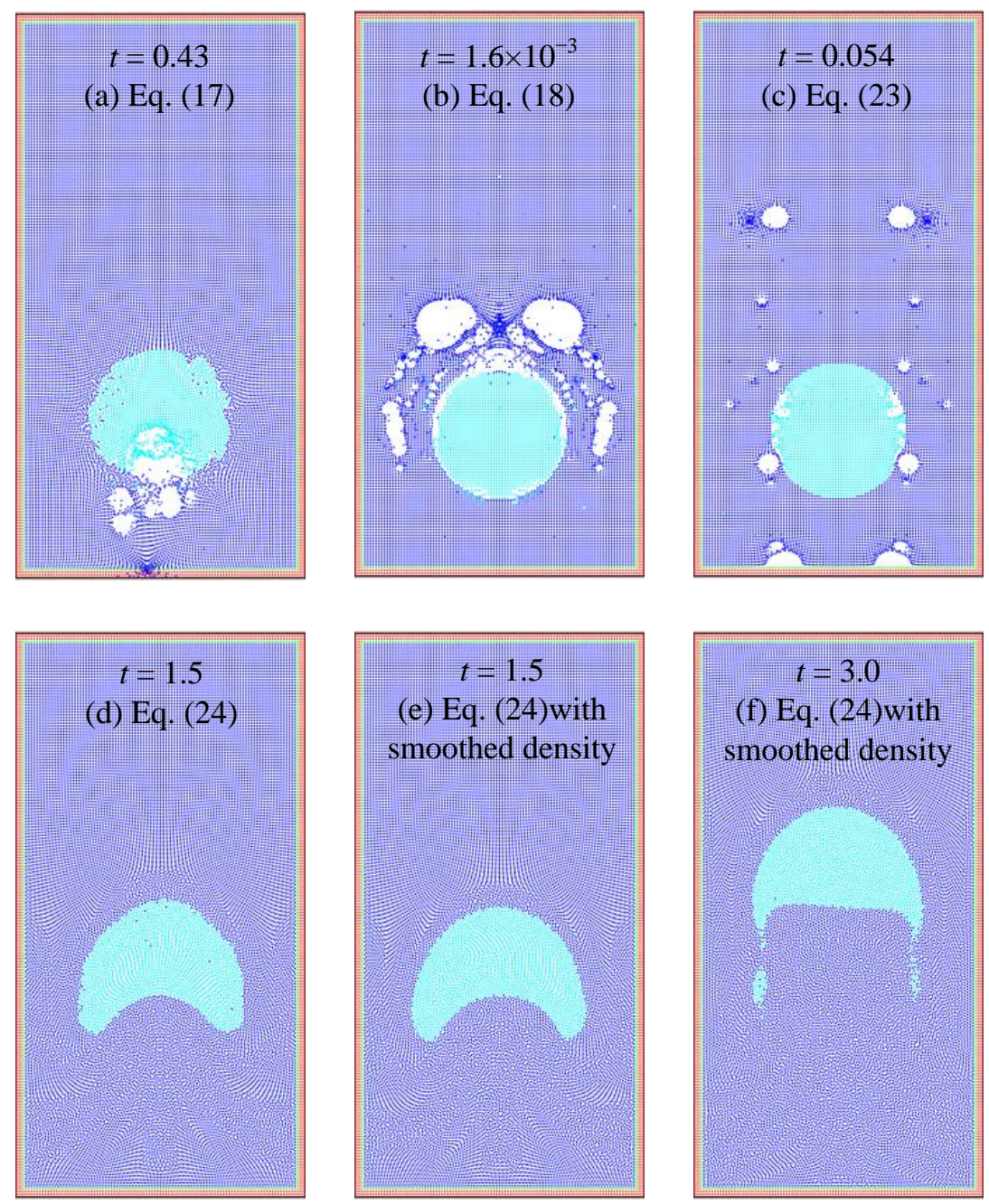

Fig. 7. Stability test of the simulated bubble rising problem (density ratio=1000:1) by different pressure gradient models using the MMPS method based on continuous acceleration. 


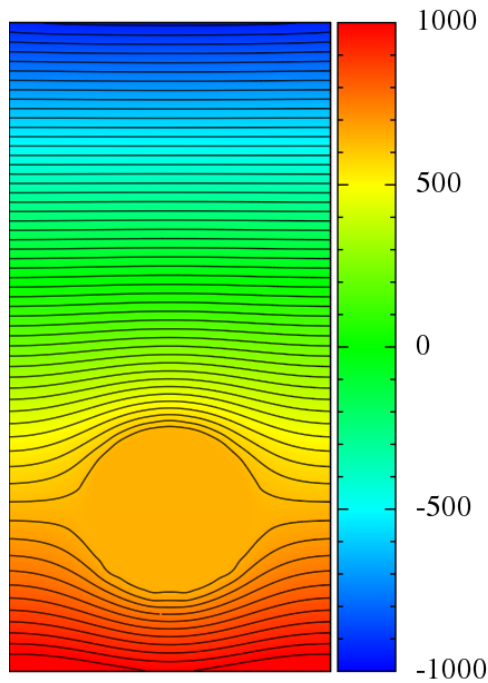

Fig. 8. Pressure profile of the simulated bubble rising problem (density ratio=1000:1) at $t=0.001 \mathrm{~s}$. The curves are contours of pressure. 


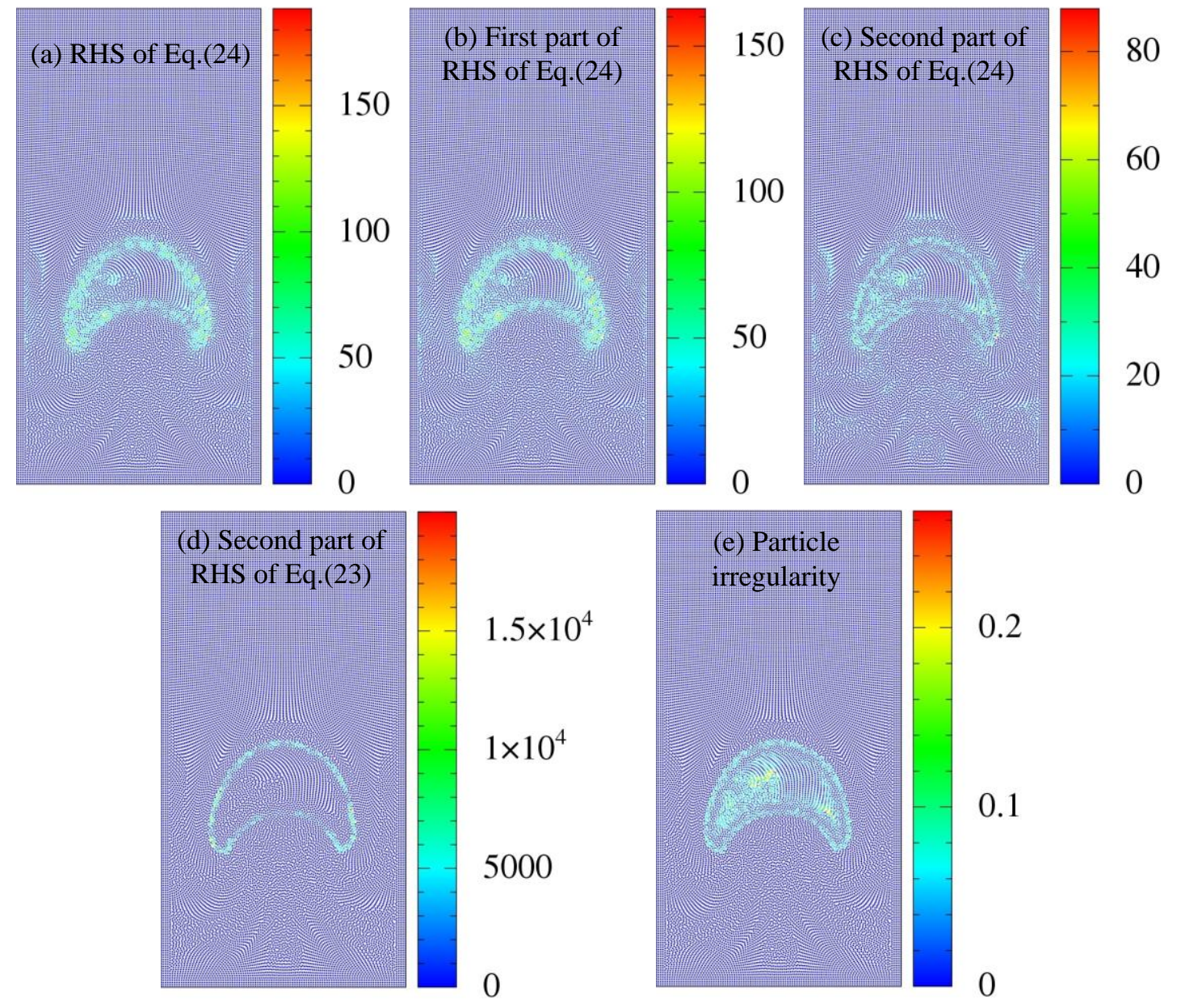

Fig. 9. The calculated accelerations by the different parts of pressure gradient models at $t=1.5 \mathrm{~s}$. 

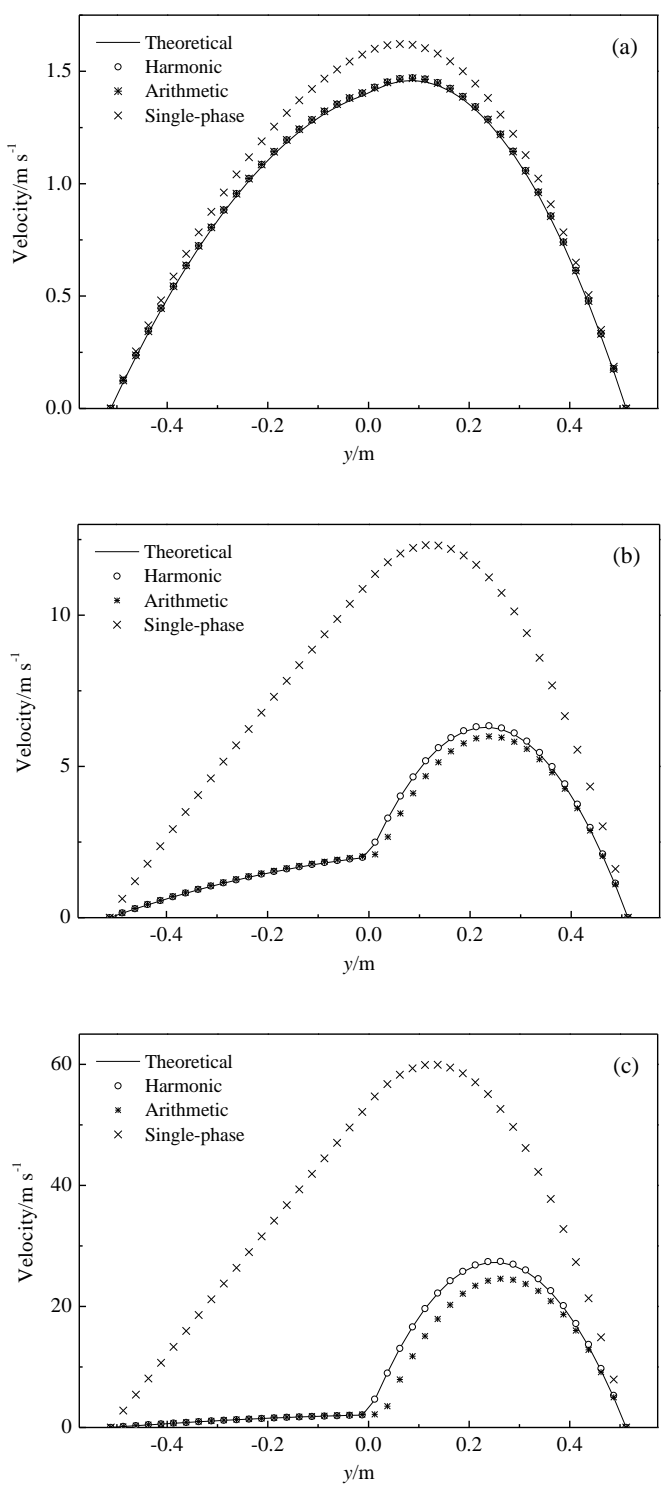

Fig. 10. Steady velocity profiles by different multi-viscosity models with the viscosity ratio of (a) $M=2$, (b) $M=20$ and (c) $M=100$. 

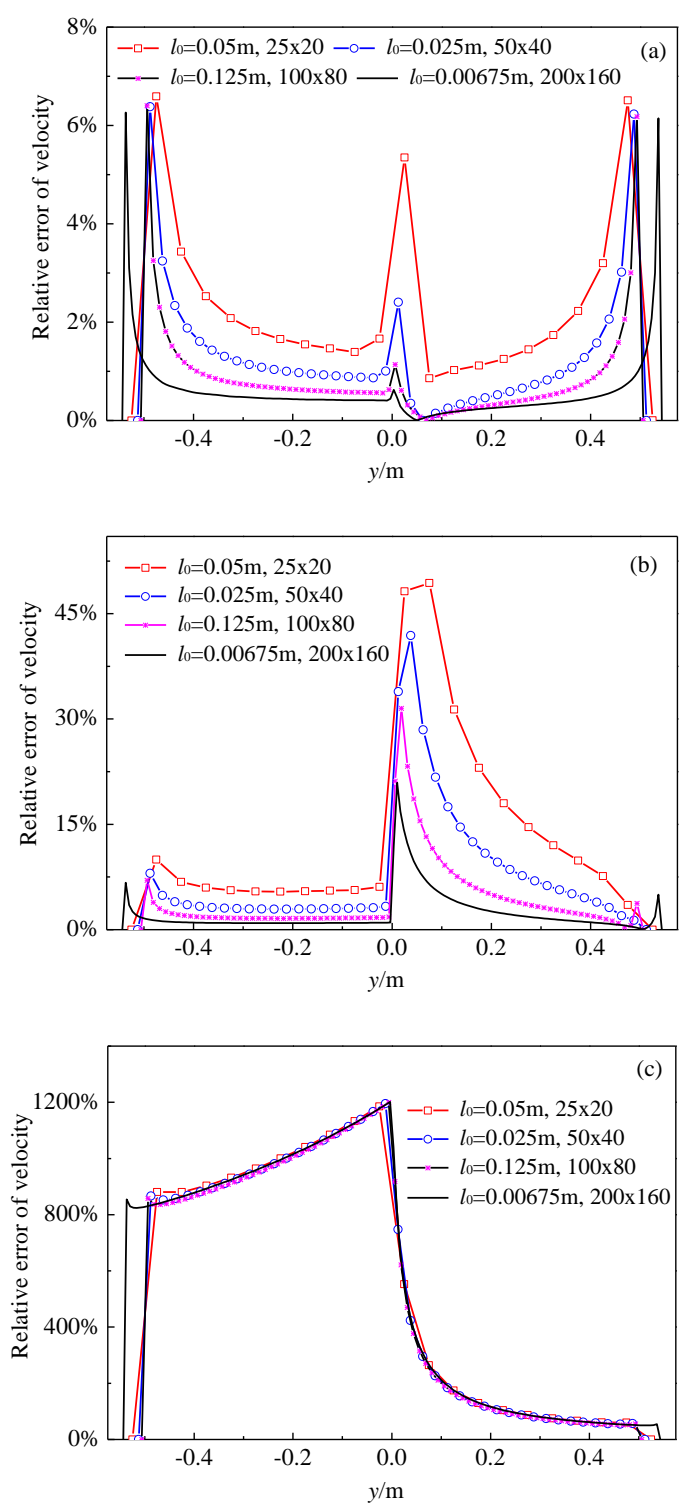

Fig. 11. Relative errors of steady velocity profiles with different particle distances of:

(a) the harmonic multi-viscosity model (i.e. Eq. (8)), (b) arithmetic multi-viscosity model and (c) single-phase viscosity model (i.e. Eq. (3)). 

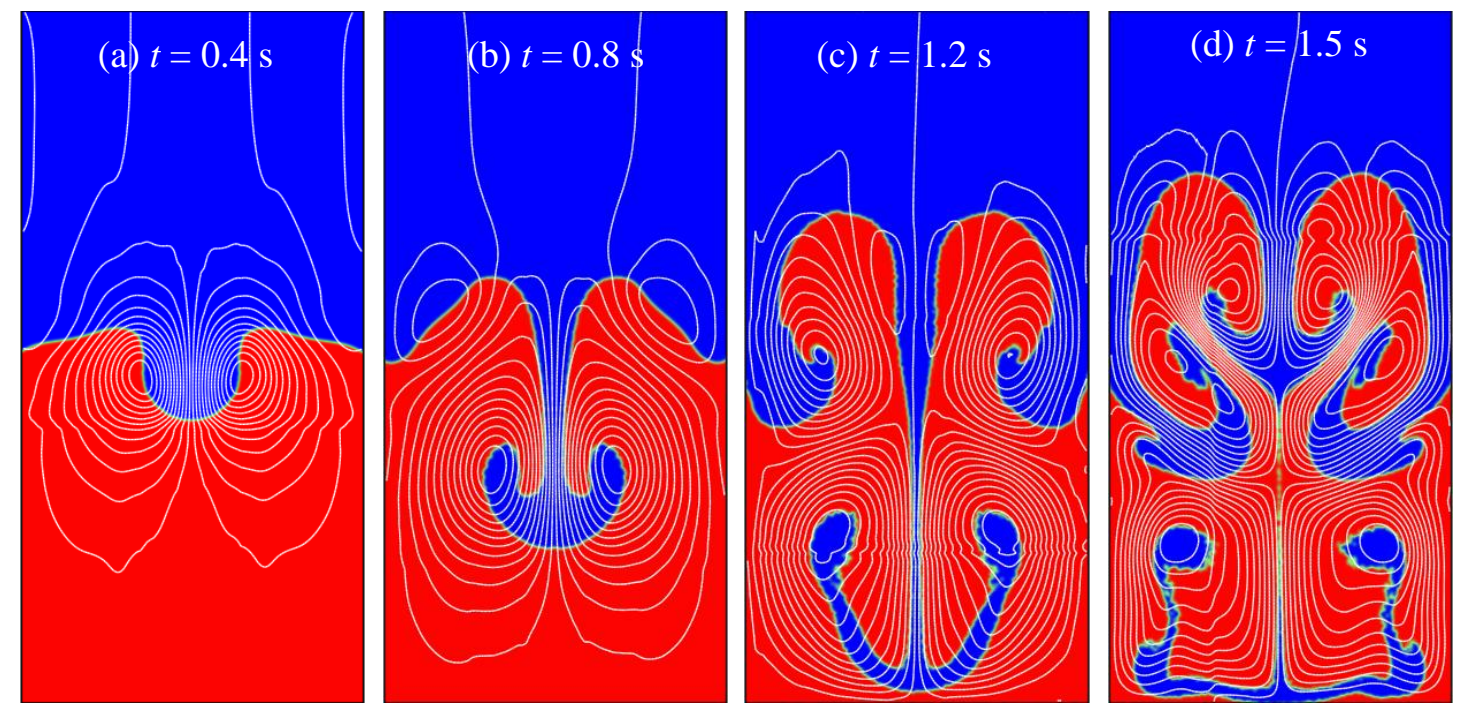

Fig. 12. Snapshots of $\mathrm{R}-\mathrm{T}$ instability with streamlines at different moments by MMPS-CA (the particle arrangement is $200 \times 400$ ). 

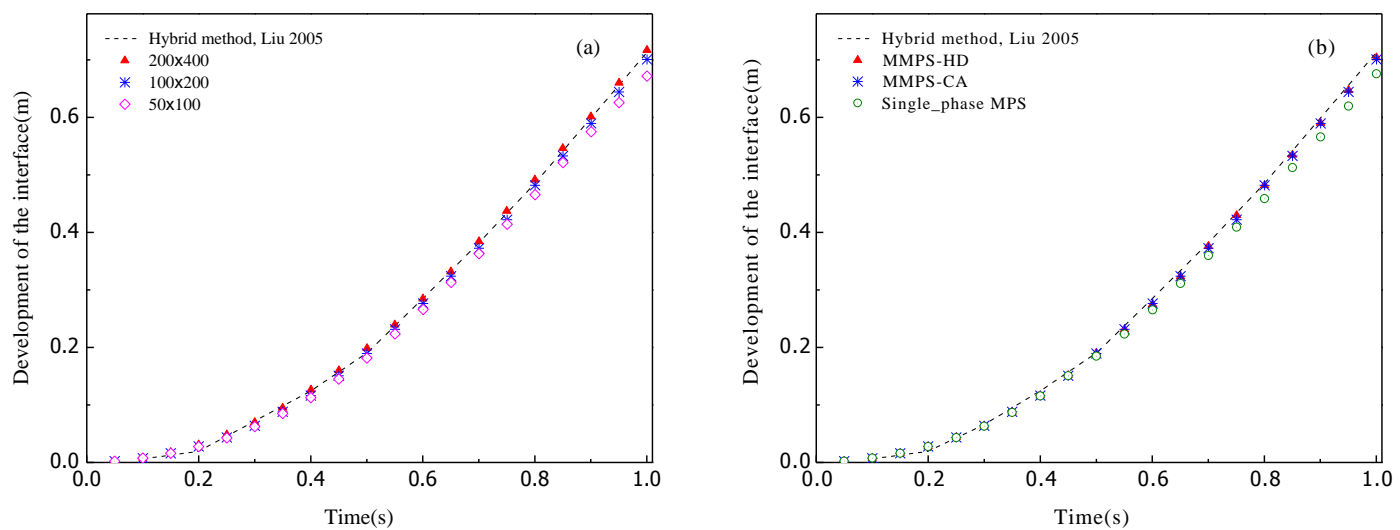

Fig. 13. Evolution of R-T instability: (a) convergence test; (b) comparison of different methods. 

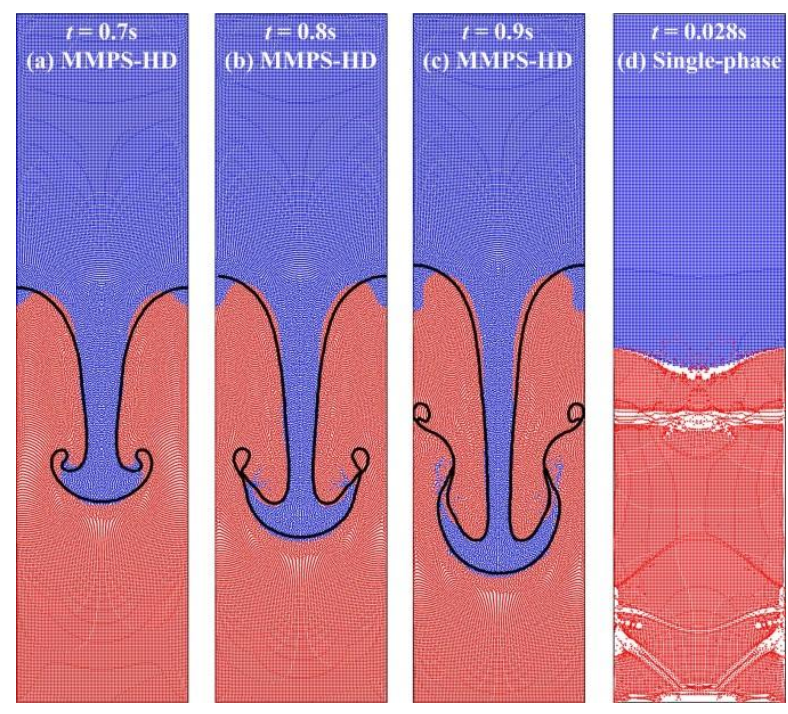

Fig. 14. Comparison of $\mathrm{R}-\mathrm{T}$ instability evolutions at different moments. The solid lines are interfaces simulated by front-tracking [58]. Figures (a) - (c) are the results simulated by MMPS-HD. Figure (d) is the result simulated by the single-phase MPS method (instability happens at $t=0.028 \mathrm{~s}$ ). 


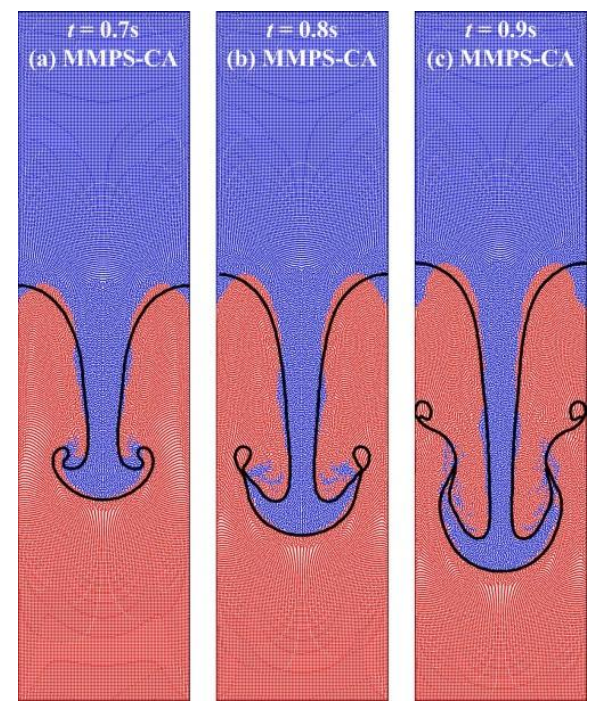

Fig. 15. Comparison of $\mathrm{R}-\mathrm{T}$ instability evolutions simulated by MMPS-CA at different moments. The solid lines are interfaces simulated by front-tracking [58]. 

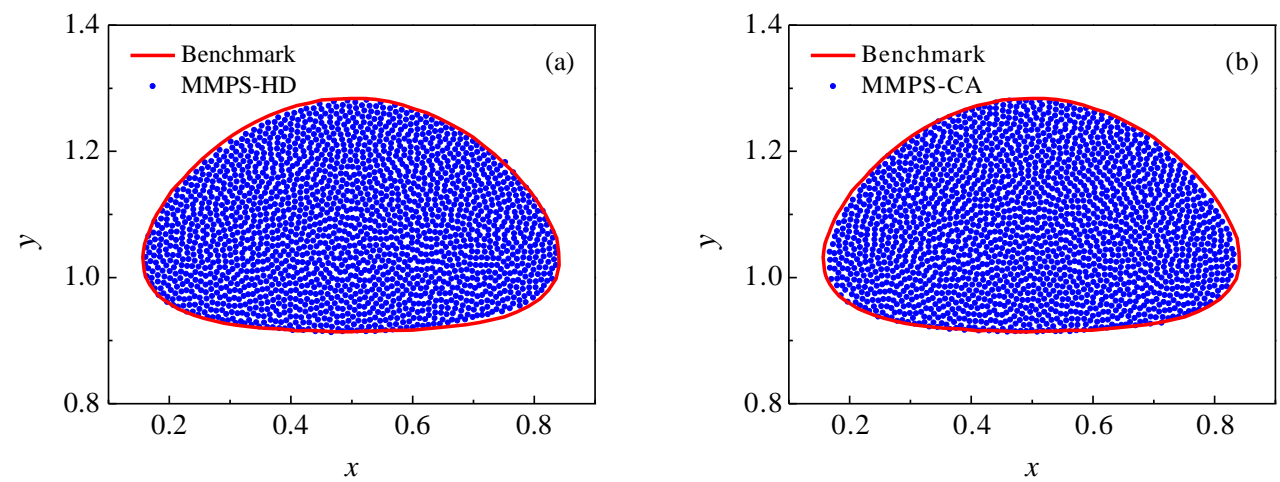

Fig. 16. Comparison of bubble shapes at $t=3$ for benchmark case 1 in $2 \mathrm{D}$. 

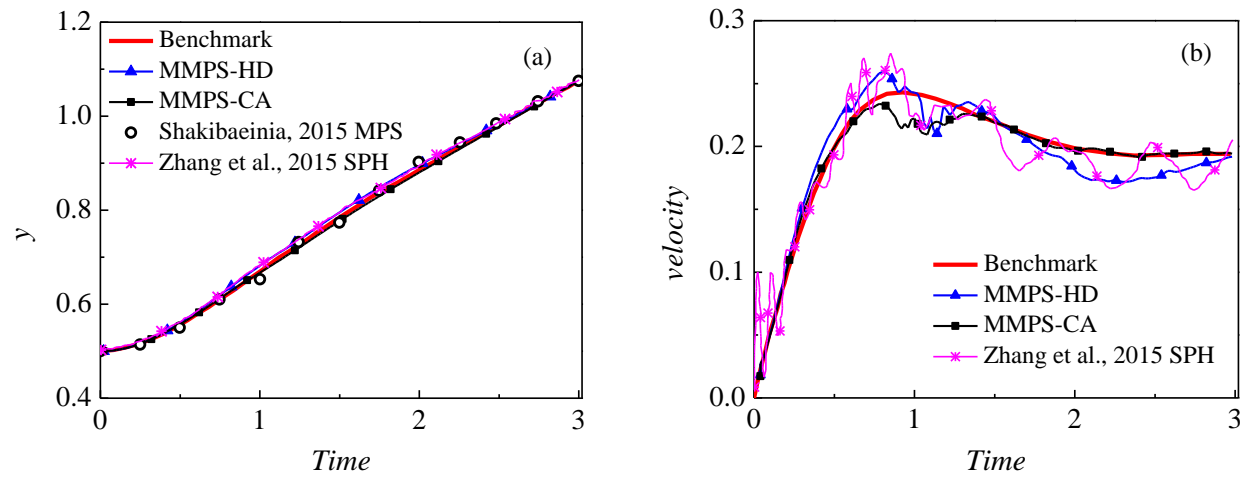

Fig. 17. Comparison of (a) centers of mass and (b) rising velocities for benchmark case 1 in $2 \mathrm{D}$. 

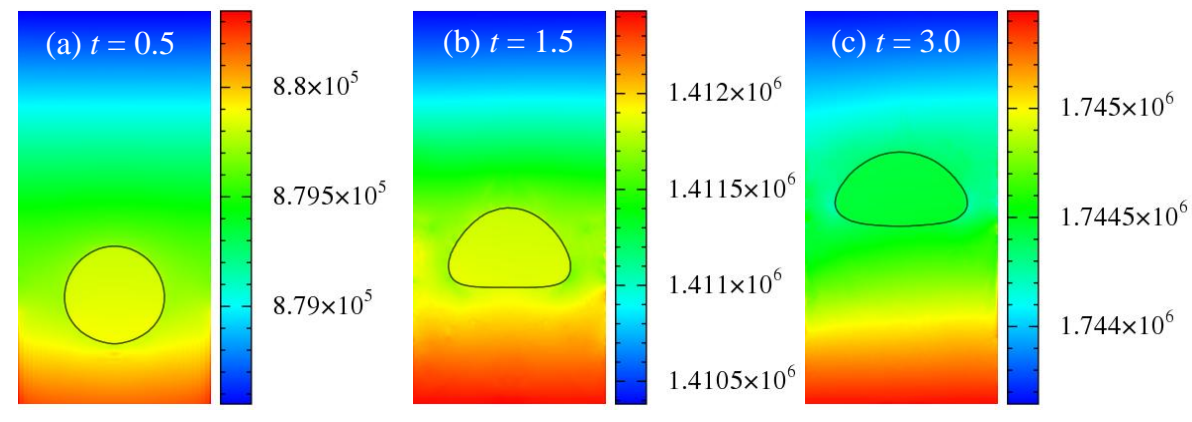

Fig. 18. The pressure profiles simulated by MMPS-CA at three typical moments in benchmark case 1 . The curve indicates the bubble shape. 

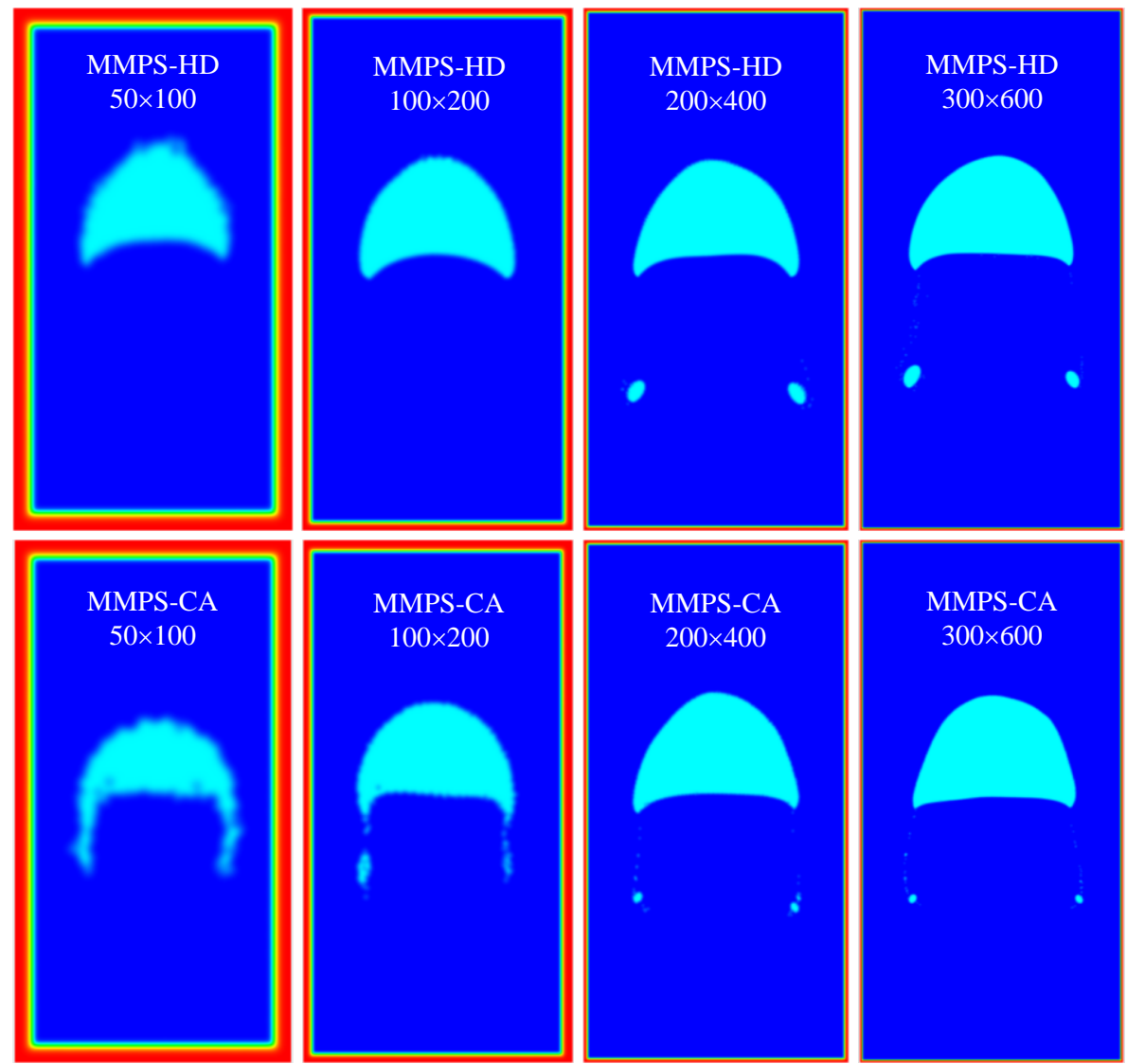

Fig. 19. Snapshots of bubble shapes simulated using different particle resolutions. 

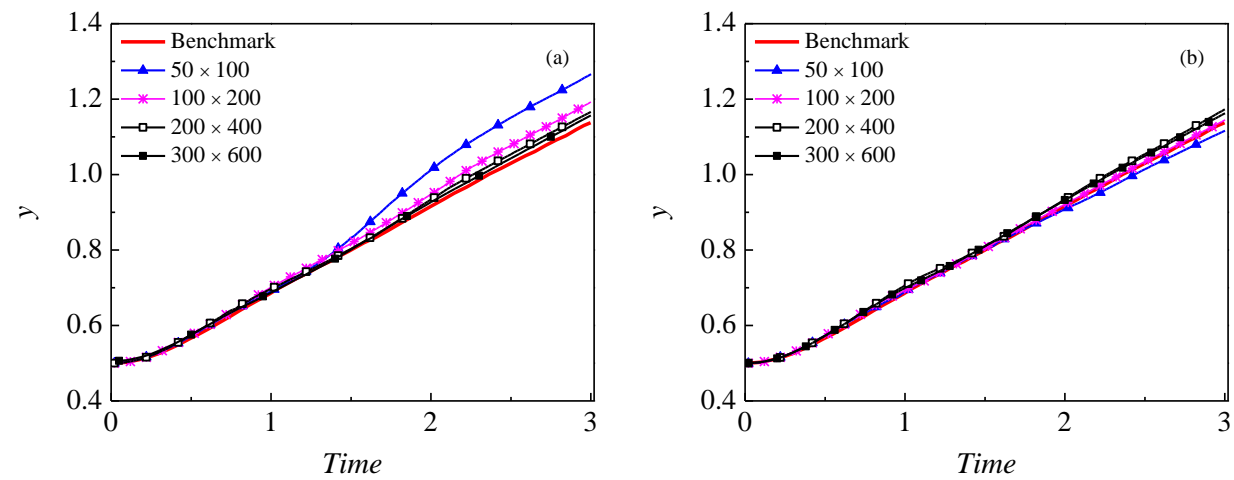

Fig. 20. Simulated evolution of centers of mass with different particle resolutions using (a) MMPS-HD and (b) MMPS-CA. 

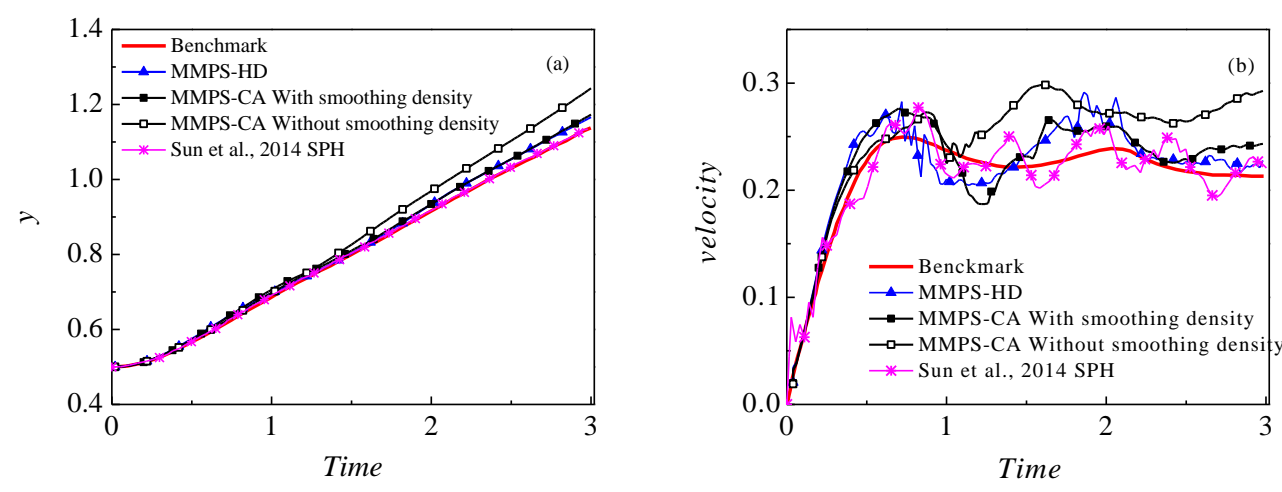

Fig. 21. Comparison of (a) centers of mass and (b) rising velocities for benchmark case 2 where density ratio $=1000$ and viscosity ratio $=100$. 

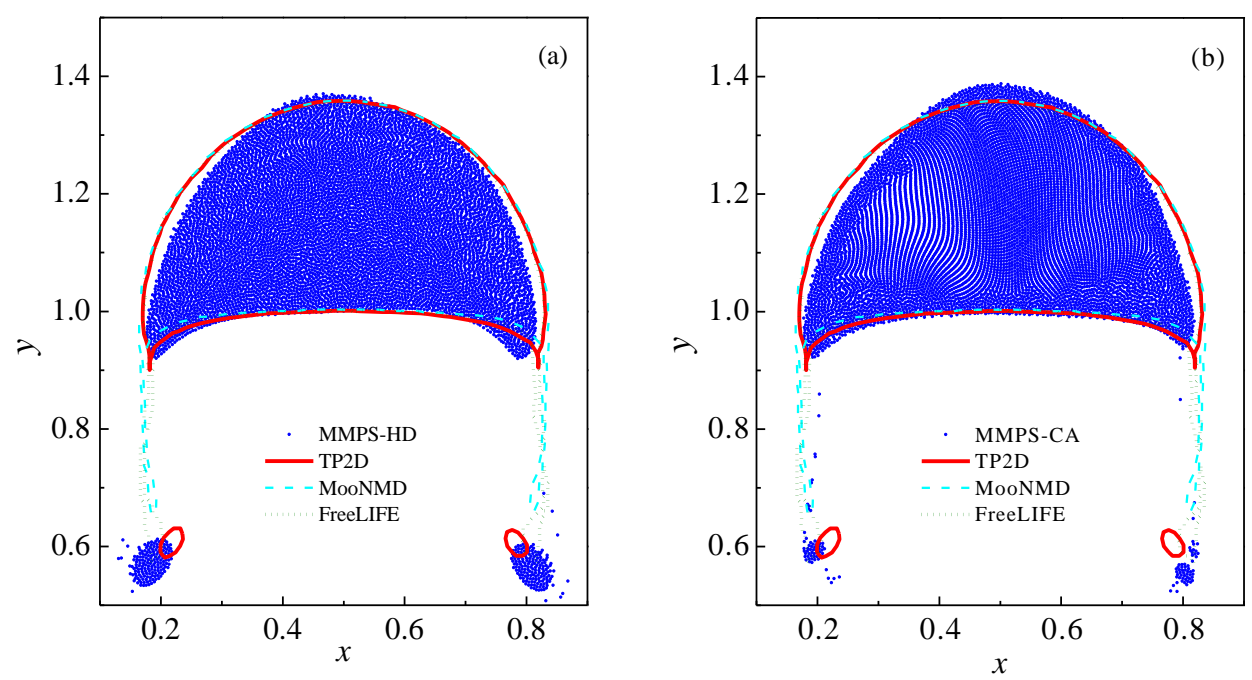

Fig. 22. Comparison of bubble shapes using $200 \times 400$ at $t=3$ for benchmark case 2 . 


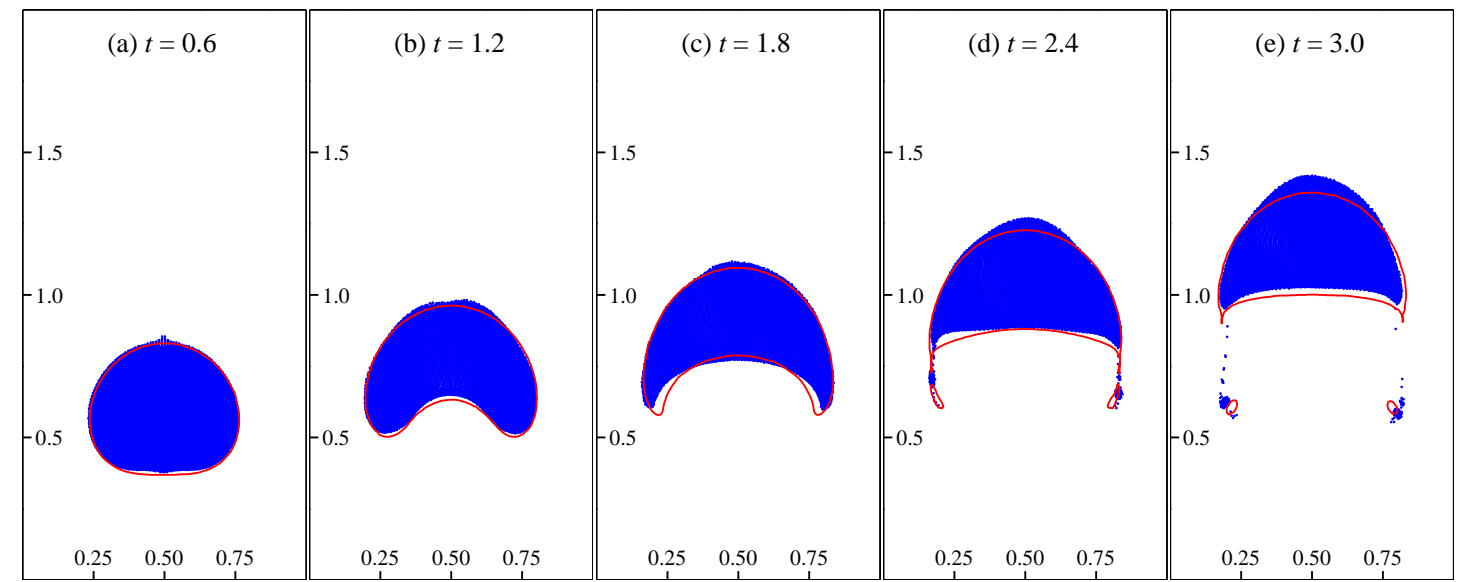

Fig. 23. Comparison between simulated bubble shapes by MMPS-CA and results by TP2D (curves) in [38] at different moments for benchmark case 2. 


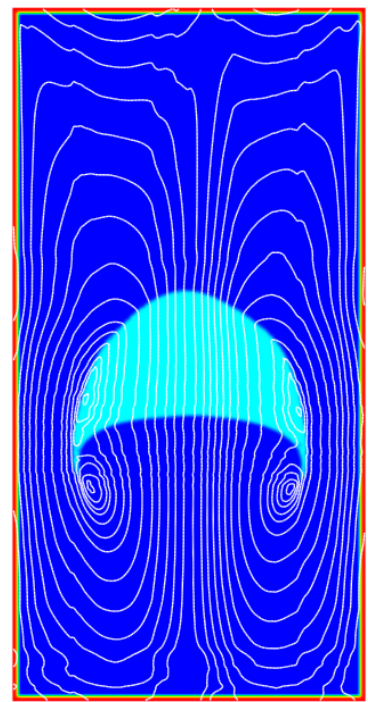

Fig. 24. Streamlines of the simulated benchmark case 2 by MMPS-CA at $t=2.1$. 

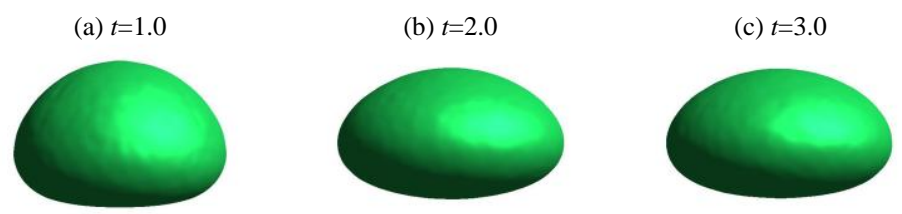

Fig. 25. Bubble shapes simulated by MMPS-CA for benchmark case 1 in 3D. 

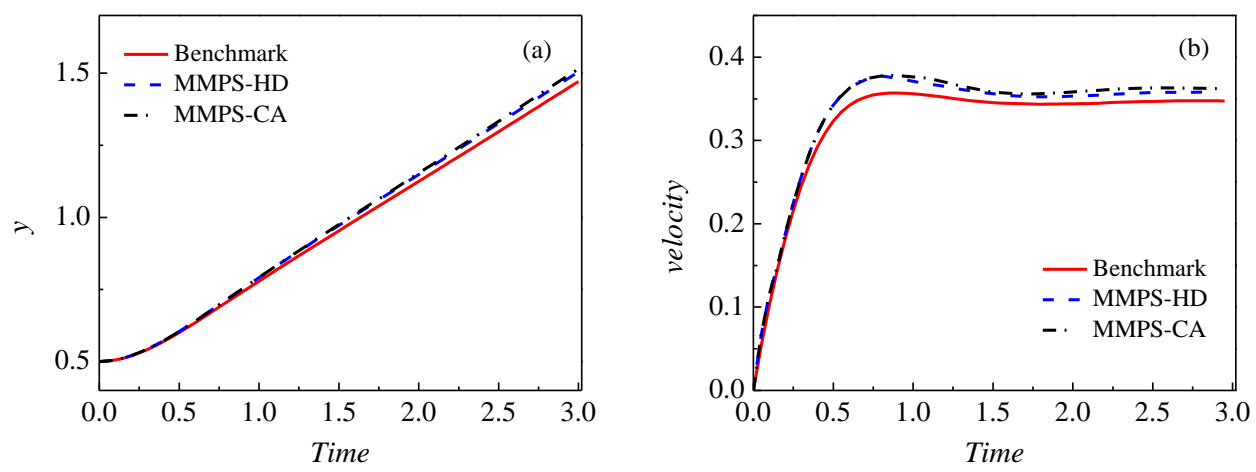

Fig. 26. Comparison of (a) centers of mass and (b) rising velocities for benchmark case 1 in $3 \mathrm{D}$. 

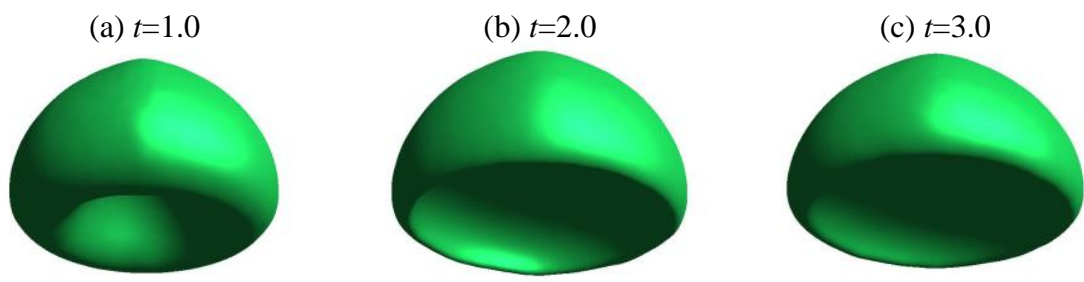

Fig. 27. Bubble shapes simulated by MMPS-HD for benchmark case 1 in 3D. 

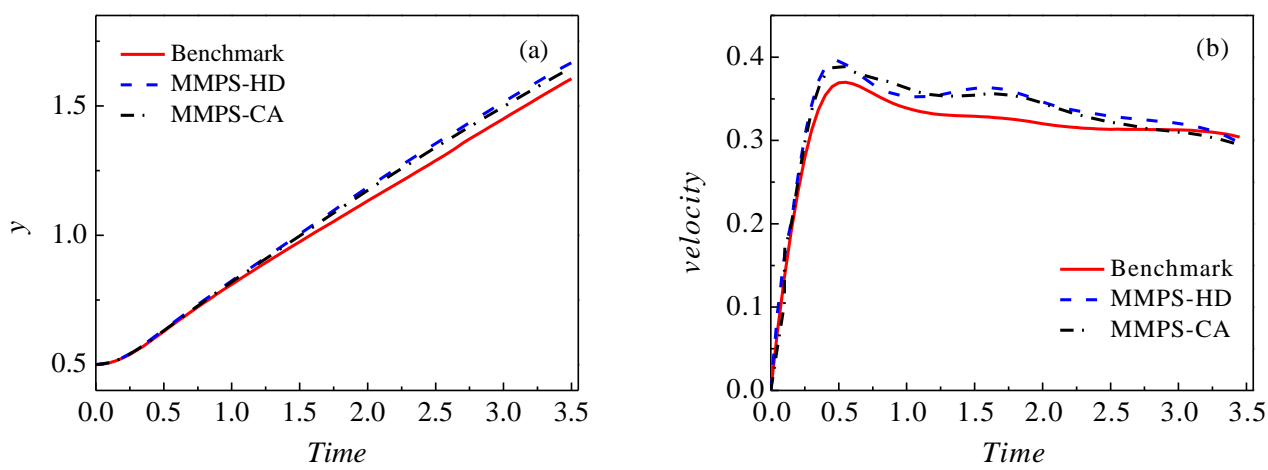

Fig. 28. Comparison of (a) centers of mass and (b) rising velocities for benchmark case 2 in 3D. 


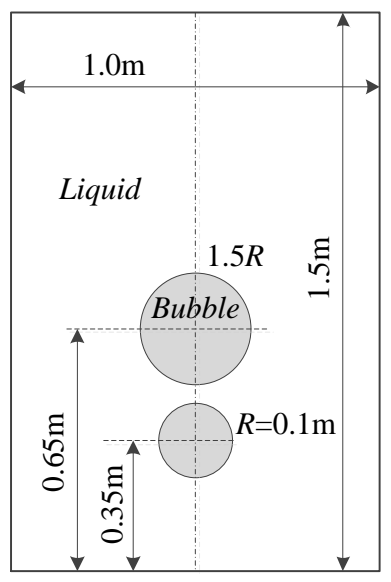

Fig. 29. Diagram of the merging of two bubbles. 

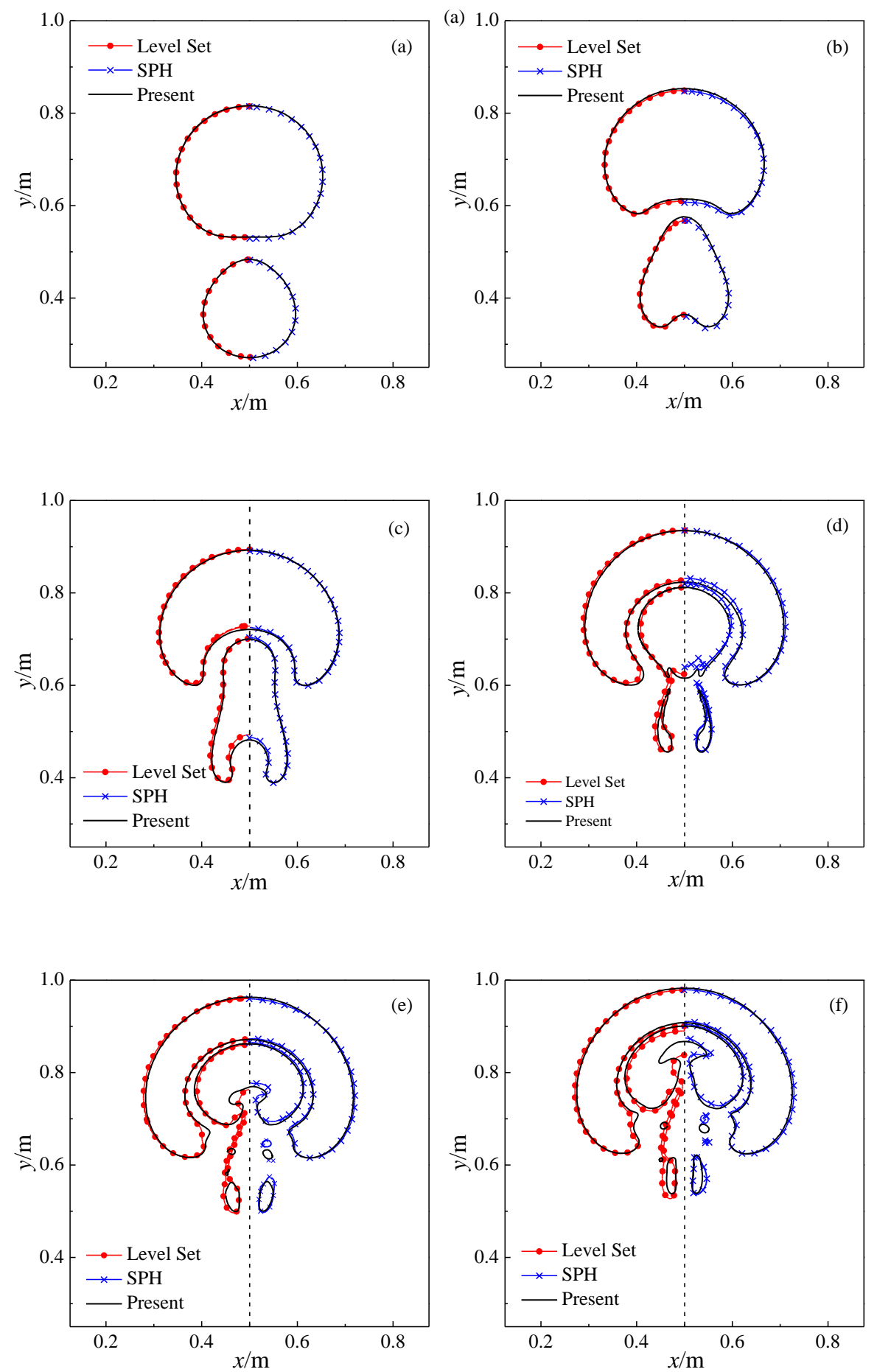

Fig. 30. Comparison of two bubbles merging with literature results [12] at different moments: (a) $t(g / R)^{1 / 2}=0.63$; (b) $t(g / R)^{1 / 2}=1.27$; (c) $t(g / R)^{1 / 2}=1.90$; (d) $t(g / R)^{1 / 2}=$ 2.53 ; (e) $t(g / R)^{1 / 2}=2.85$; (f) $t(g / R)^{1 / 2}=3.16$. 


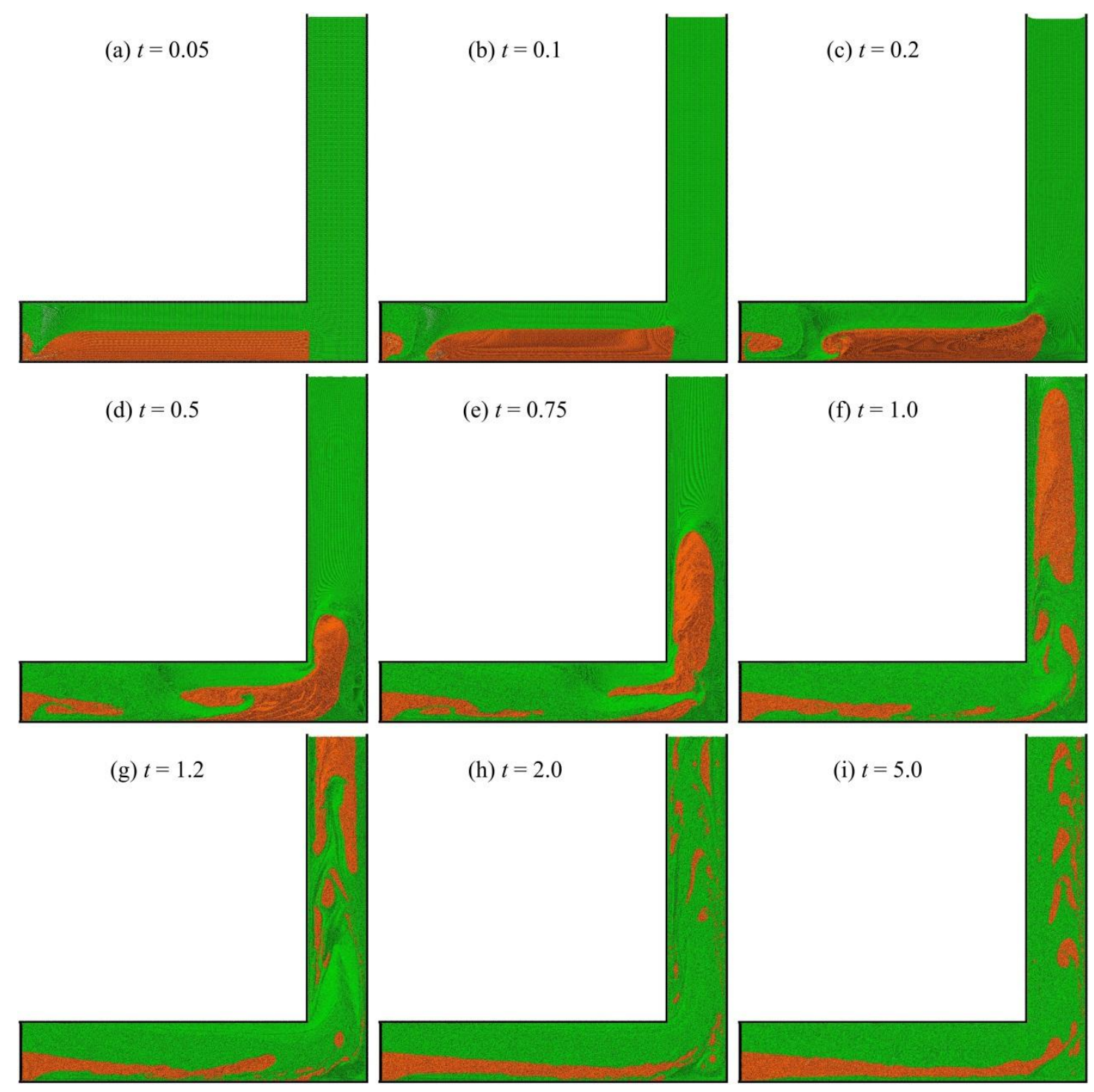

Fig. 31. Snapshots of simulated bubble rising in a right-angle tube with complex breakup behaviors (density ratio $=1000$; viscosity ratio $=100$ ). 

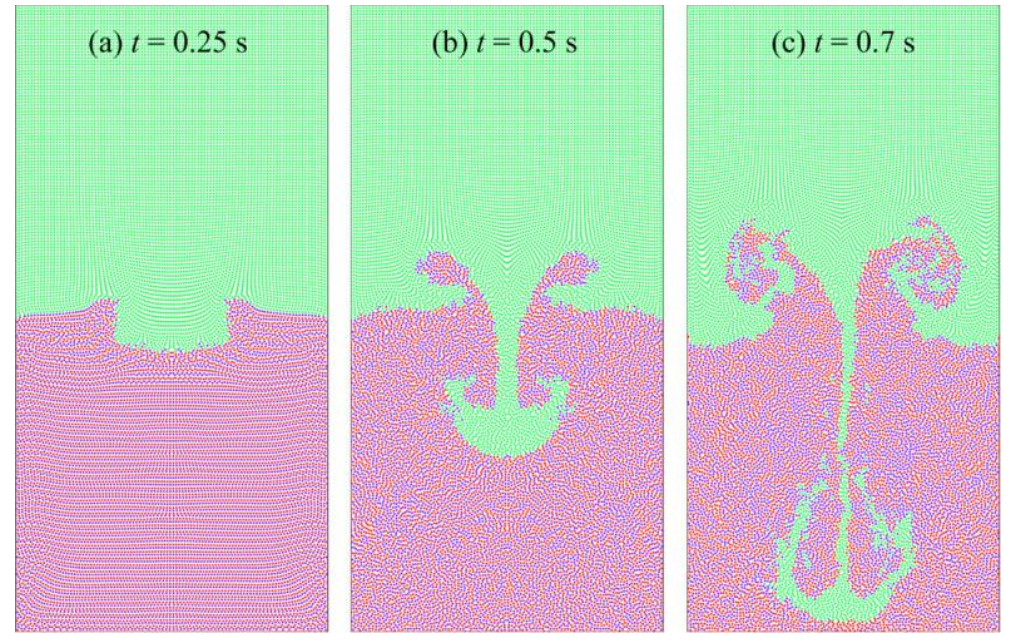

Fig. 32. $\mathrm{R}-\mathrm{T}$ instability of a continuous phase and a bubbly phase at three selected moments (the largest density ratio is 500 and no density smoothening). 